TRANSACTIONS OF THE

AMERICAN MATHEMATICAL SOCIETY

Volume 352, Number 5, Pages 1953-1983

S 0002-9947(99)02539-8

Article electronically published on December 10, 1999

\title{
LACUNARITY OF SELF-SIMILAR AND STOCHASTICALLY SELF-SIMILAR SETS
}

\author{
DIMITRIS GATZOURAS
}

\begin{abstract}
Let $K$ be a self-similar set in $\mathbb{R}^{d}$, of Hausdorff dimension $D$, and denote by $|K(\epsilon)|$ the $d$-dimensional Lebesgue measure of its $\epsilon$-neighborhood. We study the limiting behavior of the quantity $\epsilon^{-(d-D)}|K(\epsilon)|$ as $\epsilon \rightarrow 0$. It turns out that this quantity does not have a limit in many interesting cases, including the usual ternary Cantor set and the Sierpinski carpet. We also study the above asymptotics for stochastically self-similar sets. The latter results then apply to zero-sets of stable bridges, which are stochastically self-similar (in the sense of the present paper), and then, more generally, to level-sets of stable processes. Specifically, it follows that, if $K_{t}$ is the zero-set of a realvalued stable process of index $\alpha \in(1,2]$, run up to time $t$, then $\epsilon^{-1 / \alpha}\left|K_{t}(\epsilon)\right|$ converges to a constant multiple of the local time at 0 , simultaneously for all $t \geq 0$, on a set of probability one.

The asymptotics for deterministic sets are obtained via the renewal theorem. The renewal theorem also yields asymptotics for the mean $\mathbb{E}[|K(\epsilon)|]$ in the random case, while the almost sure asymptotics in this case are obtained via an analogue of the renewal theorem for branching random walks.
\end{abstract}

\section{INTRODUCTION}

Consider the following construction of a Cantor set on the real line. Start with the unit interval $I=[0,1]$ and divide it into $n \geq 3$ equal subintervals, each of length $r=n^{-1}$. Let $N$ be an integer with $1<N<n$ and keep any $N$ of the $n$ subintervals $I_{i}=\left[i n^{-1},(i+1) n^{-1}\right]$, say $I_{i_{1}}, \ldots, I_{i_{N}}$, and discard the remaining ones. Next divide each of the intervals $I_{i_{k}}$ kept in the first step into $n$ equal sub-subintervals, of length $r^{2}=n^{-2}$ now, and keep only $N$ of these within each $I_{i_{k}}$, always according to the same pattern as in the first step of the construction. Continuing ad infinitum, this construction leads to a Cantor set $K$ on the line, of Hausdorff and Minkowski (or box) dimension equal to $\log _{n} N$. Alternatively, $K=\left\{\sum_{1}^{\infty} x_{j} n^{-j}: x_{j} \in\left\{i_{1}, \ldots, i_{N}\right\}\right\}$.

Now consider two particular cases, both with $r=1 / 16$ and $N=4$, but different patterns. For the first construction keep the two leftmost and two rightmost of the subintervals in each step. For the second construction use the pattern in which the four subintervals kept are uniformly spread out; i.e., we keep the $1^{\text {st }}, 6^{\text {th }}, 11^{\text {th }}$ and $16^{\text {th }}$. In both constructions the limit set has dimension $D=\frac{1}{2}$ but according

Received by the editors September 8, 1998 and, in revised form, March 4, 1999.

2000 Mathematics Subject Classification. Primary 28A80, 28A75, 60D05; Secondary 60K05, $60 \mathrm{G} 52$.

Key words and phrases. Cantor set, $\epsilon$-neighborhood, Minkowski content, branching random walk, renewal theorem, stable process. 

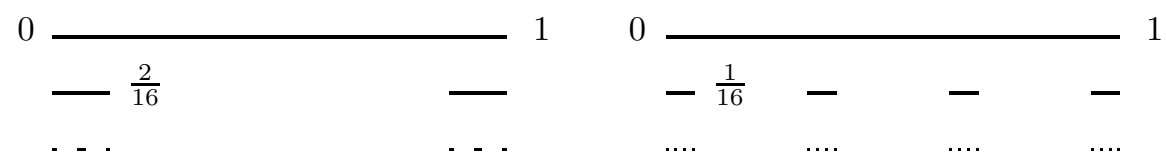

Figure 1. Two Cantor sets with $D=\frac{1}{2}$.

to Mandelbrot ([19], page 313) these two limit sets look quite different (see Figure 1): the outcome of the first construction looks like a 'few points', hence 'mimics the dimension $D=0$ ', while the outcome of the second construction looks like a 'full interval' and hence 'mimics the dimension $D=1$ '. He calls the set of the first construction a set of 'high lacunarity' and the set of the second construction a set of 'low lacunarity'.

In an attempt to give a numerical measure for lacunarity Mandelbrot considers the length of the $\epsilon$-neighborhood, $|K(\epsilon)|$, of the limit set $K$. It is well known that $|K(\epsilon)| \approx \epsilon^{1-D}$, in the sense that

$$
\lim _{\epsilon \rightarrow 0} \frac{\log |K(\epsilon)|}{\log \epsilon}=1-D,
$$

giving the Minkowski or box dimension $D$ of $K$. Mandelbrot observes that in 'many' cases one has the stronger relation $|K(\epsilon)| \sim L \epsilon^{1-D}$, in the sense that the ratio of the two sides tends to 1 as $\epsilon \rightarrow 0$; he calls $L$ the 'prefactor' and proposes $L^{-1}$ as a numerical measure of lacunarity, provided of course it exists.

To put it in a different way: the asymptotic ratio of the logarithms of $|K(\epsilon)|$ and $\epsilon$ gives some crude information about $K$, relating only to its dimension; by considering the more delicate direct ratio $|K(\epsilon)| / \epsilon^{1-D}$ one hopes to capture more refined information about $K$, also relating to its finer topological structure.

In this paper we give sufficient conditions for $L$ to exist, i.e., for $\epsilon^{-(d-D)}|K(\epsilon)|$ to converge to a limit as $\epsilon \rightarrow 0$, for self-similar sets $K \subset \mathbb{R}^{d}$; by self-similar here we mean both strictly self-similar and stochastically self-similar sets, i.e., random sets which are only self-similar at the level of distributions. As a matter of fact, for such $K$, we obtain exact asymptotics for $|K(\epsilon)|$ as $\epsilon \rightarrow 0$. It turns out that $\lim _{\epsilon \rightarrow 0} \epsilon^{-(d-D)}|K(\epsilon)|$ does not exist in many interesting cases, like for example the usual ternary Cantor set (see section 4.1). However, the limit $\lim _{T \rightarrow \infty} T^{-1} \int_{0}^{T} e^{(d-D) t}\left|K\left(e^{-t}\right)\right| d t$ always exists for self-similar sets and one may use this quantity instead as a measure of lacunarity, if one is to summarize this information into a single number (compare with Bedford and Fisher [1]). We stress that our aim is to prove results on existence; the question of whether the proposed quantities are 'good measures of lacunarity' is a different issue, requiring separate investigation.

We remark in passing that the asymptotics of $|K(\epsilon)|$ are also directly related to the concept of 'Minkowski measurability'. A compact set $K \subset \mathbb{R}^{d}$, of Minkowski dimension $D$, is Minkowski measurable precisely when $\epsilon^{-(d-D)}|K(\epsilon)|$ has a limit $L \in(0, \infty)$, as $\epsilon \rightarrow 0 ; L$ is then the Minkowski content of $K$ in this context. (Thus Mandelbrot defines lacunarity as the reciprocal of Minkowski content, when this is defined.) Minkowski measurability and Minkowski content have attracted interest in recent years, because of the central role they play in a conjecture of Lapidus ([14]), and related work of Lapidus and Pomerance ([15], [16]), pertaining to the 
Weyl-Berry conjecture on the distribution of the eigenvalues of the Laplacian, on domains with 'fractal' boundaries 1$]$

Our main tool for studying the aforementioned asymptotics for $|K(\epsilon)|$ is the renewal theorem from probability theory and one of the objectives of this paper (besides obtaining the results themselves) is to emphasize the suitability of renewal theory as a tool in the study of self-similar sets. The first application of the (standard) renewal theorem to (strictly) self-similar sets is due to Lalley, who applied it in [12 to obtain the exact asymptotics for the packing and covering functions of such sets. Later, in [13], he used similar methods for fractals with some approximate self-similarity, such as limit sets of Kleinian groups; for the results of the latter paper he actually had to develop suitable analogues of the standard renewal theorem. We stress the fact that in both papers of Lalley mentioned above and also in section 2 of the present paper, pertaining to strictly self-similar sets, the renewal theorem is applied to study deterministic sets. One can use similar methods for stochastically self-similar sets and this is done in section 3 below. Results which hold in mean, for such random sets, are obtained by use of the ordinary renewal theorem, while almost sure results are obtained via an analogue of the renewal theorem for branching random walks, due to Nerman ([22]).

The rest of the paper is organized as follows. In section 2 we deal with strictly self-similar sets; the main results there are Theorem 2.3 and its companion, Theorem 2.4. In section 3 we deal with stochastically self-similar sets. In particular, in subsection 3.1 we describe the kind of random sets we are considering and state the main results concerning them, Theorems 3.1 and 3.2 In subsection 3.2 we state Nerman's theorem in the language of branching random walks and in 3.3 we give the proof of Theorem 3.1. Subsection 3.4 deals with 'mean convergence' and in subsection 3.5 we prove Theorem 3.2 Finally, in section 4 we discuss some examples and applications. One of them pertains to level-sets of stable bridges and stable processes, for which $\epsilon^{-(d-D)}\left|K_{t}(\epsilon)\right|$ converges and the limit is local time at 0 ; here $K_{t}$ is the zero-set of the process, run up to time $t$, and the convergence holds simultaneously for all $t \geq 0$, on a set of probability one (Theorem 4.1). We thus reprove a result which follows directly from results of Fristedt and Taylor ([9]). (Our proof, however, does not assume a priori existence of local time and we thus obtain local time as originally envisaged by P. Levy.)

After proving Theorem 2.3 (deterministic sets), we learned of Falconer's paper 7], which also contains versions of Theorem 2.3 (i), but with the additional assumption that $K$ is totally disconnected. He also uses renewal theorems, but his approach is, to a certain extent, different than ours. We decided to include Theorem 2.3 here because: 1) it covers basic examples, like e.g. the von Koch snowflake or the Sierpinski carpet, which Falconer's result does not; 2) its proof conveys the basic idea of the approach better, as it lacks certain technicalities which are present in the random case.

Acknowledgments. The author wishes to thank B. Mandelbrot, Y. Peres, who drew his attention to the problem, by pointing out that $\epsilon^{-(1-D)}|K(\epsilon)|$ does not have a

\footnotetext{
${ }^{1}$ Note added in proof: After submitting the final version of this paper we also learned of 26]; conjectures 3 and $3 \mathrm{r}$ there relate directly to the results in this paper and the conjecture in section 5 .
} 
limit, as $\epsilon \rightarrow 0$, for the usual ternary Cantor set, and S. Lalley, who had independently obtained a renewal theorem for branching random walks, and from whom the author first heard about such a theorem.

\section{STRICTLY SELF-SIMILAR SETS}

2.1. Background and statement of results. Let $\left\{\phi_{1}, \ldots, \phi_{N}\right\}$ be a finite set of similarities of $\mathbb{R}^{d}$; i.e., each $\phi_{i}$ satisfies

$$
\left|\phi_{i}(x)-\phi_{i}(y)\right|=r_{i}|x-y| \quad \forall x, y \in \mathbb{R}^{d},
$$

for some constant $r_{i}$. Assume $0<r_{i}<1$, for each $i \in\{1, \ldots, N\}$, i.e., each $\phi_{i}$ is a contraction, and let $D$ be the unique real number for which

$$
\sum_{i=1}^{N} r_{i}^{D}=1
$$

A well known result of Hutchinson ([10]) asserts that there exists a unique nonempty compact subset $K$ of $\mathbb{R}^{d}$ which is invariant with respect to $\left\{\phi_{1}, \ldots, \phi_{N}\right\}$, i.e.,

$$
K=\bigcup_{i=1}^{N} \phi_{i}(K) .
$$

The set $K$ is then a (strictly) self-similar set and is in many cases a 'fractal'. The examples in the introduction, which include the usual ternary Cantor set, the von Koch snowflake and the Sierpinski carpet, all arise in this manner (see [10]). We may, for example, obtain the ternary Cantor set by taking $\phi_{1}, \phi_{2}: \mathbb{R} \rightarrow$ $\mathbb{R}, \phi_{1}(x)=x / 3, \phi_{2}(x)=x / 3+2 / 3$. For the von Koch snowflake we may take $\phi_{1}, \ldots, \phi_{4}: \mathbb{R}^{2} \rightarrow \mathbb{R}^{2}$, where $\phi_{i}$ is the unique similarity, with positive determinant, mapping the interval $A_{1} A_{5}$ to $A_{i} A_{i+1}$ in Figure 2. In both cases, $K$ may then be visualized as (formally, the limit is in the Hausdorff metric)

$$
K=\lim _{n \rightarrow \infty} \bigcup_{i_{1}, \ldots, i_{n}} \phi_{i_{1}} \circ \ldots \circ \phi_{i_{n}}([0,1]) .
$$

Given a set of contracting similarities $\left\{\phi_{1}, \ldots, \phi_{N}\right\}$, one says that $\left\{\phi_{1}, \ldots, \phi_{N}\right\}$ (or the corresponding invariant set $K$ ) satisfies the open set condition, if there exists a bounded open set $G$ in $\mathbb{R}^{d}$ with the properties

$\phi_{i}(G) \subseteq G$ for each $i \in\{1, \ldots, N\}$;

$\phi_{i}(G) \cap \phi_{j}(G)=\emptyset$ whenever $i \neq j$.

It is well known (see [10], [12, [21]) that if $\left\{\phi_{1}, \ldots, \phi_{N}\right\}$ are contracting similarities, with contraction ratios $r_{1}, \ldots, r_{N}$ respectively, satisfying the open set condition, then the corresponding invariant set $K$ has both Hausdorff and Minkowski dimensions equal to the unique solution $D$ of equation (2.1); this $D$ is also referred

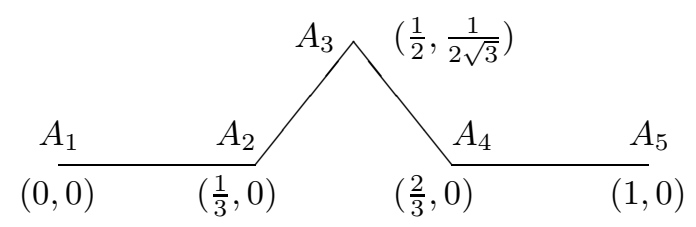

FiguRE 2. One step in the construction of the von Koch snowflake. 
to as the similarity dimension of $K$. Furthermore, $0<\mathcal{H}_{D}(K)<\infty$, where $\mathcal{H}_{D}$ denotes $D$-dimensional Hausdorff measure.

Notation 2.1. Given an arbitrary compact set $F \subset \mathbb{R}^{d}$ we will denote by $F(\epsilon)$ its $\epsilon$-neighborhood,

$$
F(\epsilon):=\left\{x \in \mathbb{R}^{d}: \operatorname{dist}(x, F)<\epsilon\right\} .
$$

Lebesgue measure - always $d$-dimensional in this paper, as the dimension of the underlying space - will be denoted by || . With these notations $|K(\epsilon)|$ is the length, area, volume, etc. of the $\epsilon$-neighborhood of $K$.

Notation 2.2. Given $\lambda>0$ we write $\lambda \mathbb{Z}:=\{\ldots,-2 \lambda,-\lambda, 0, \lambda, 2 \lambda, \ldots\}$.

It is well known that

$$
\lim _{\epsilon \rightarrow 0} \frac{\log |K(\epsilon)|}{\log \epsilon}=d-D ;
$$

here $D$ should be regarded as the Minkowski dimension of $K$. The following theorem is a refinement of this.

Theorem 2.3. Let $\phi_{1}, \ldots, \phi_{N}: \mathbb{R}^{d} \rightarrow \mathbb{R}^{d}$ be contracting similarities, with similarity ratios $r_{1}, \ldots, r_{N} \in(0,1)$, and let $K$ be the corresponding invariant set (2.2). Assume that $\left\{\phi_{1}, \ldots, \phi_{N}\right\}$ satisfies the open set condition and let $D$ satisfy $\sum_{i=1}^{N} r_{i}^{D}=1$.

(i) If the numbers $\log r_{1}, \ldots, \log r_{N}$ do not concentrate on an additive subgroup of $\mathbb{R}$ of the form $\lambda \mathbb{Z}$ for some $\lambda>0$, then the following limit exists and is finite:

$$
L:=\lim _{\epsilon \rightarrow 0} \frac{|K(\epsilon)|}{\epsilon^{d-D}} .
$$

(ii) The following limit always exists and is finite:

$$
L:=\lim _{T \rightarrow \infty} \frac{1}{T} \int_{0}^{T} \frac{\left|K\left(e^{-t}\right)\right|}{e^{-t(d-D)}} d t .
$$

Observe that the ternary Cantor set, the von Koch snowflake and the Sierpinski carpet are all excluded from (i).

Note. The number $L$ is always defined, by (ii); when the limit in (i) exists it necessarily equals $L$.

Theorem 2.4. $L>0$.

We defer the proof of Theorem 2.4 to section 2.4.

2.2. Proof of Theorem 2.3. Let $K_{i}=\phi_{i}(K)$, for $i \in\{1, \ldots, N\}$, and observe that

$$
\phi_{i}(K(\epsilon))=K_{i}\left(\epsilon r_{i}\right) ;
$$

here $K_{i}\left(\epsilon r_{i}\right)$ is the $\epsilon r_{i}$-neighborhood of $K_{i}=\phi_{i}(K)$. Since each $\phi_{i}$ is a similarity,

$$
r_{i}^{d}|K(\epsilon)|=\left|\phi_{i}(K(\epsilon))\right|=\left|K_{i}\left(\epsilon r_{i}\right)\right|
$$

or equivalently,

$$
r_{i}^{d}\left|K\left(\frac{\epsilon}{r_{i}}\right)\right|=\left|K_{i}(\epsilon)\right|,
$$


for any $\epsilon>0$ and any $i \in\{1, \ldots, N\}$. Let

$$
R(\epsilon)=|K(\epsilon)|-\sum_{i=1}^{N}\left|K_{i}(\epsilon)\right| ;
$$

we then have that

$$
\begin{aligned}
|K(\epsilon)| & =\sum_{i=1}^{N}\left|K_{i}(\epsilon)\right|+R(\epsilon) \\
& =\sum_{i=1}^{N} r_{i}^{d}\left|K\left(\epsilon r_{i}^{-1}\right)\right|+R(\epsilon) .
\end{aligned}
$$

Notice here that in the totally disconnected case, i.e., when $K_{i} \cap K_{j}=\emptyset$ whenever $i \neq j$, there exists a $\delta>0$ such that $\operatorname{dist}\left(K_{i}, K_{j}\right)>\delta$ for $i \neq j$, and so $R(\epsilon)=0$ for all sufficiently small $\epsilon$. This is not true in the general case however, when distinct $K_{i}$ may intersect.

Set

$$
w(t)= \begin{cases}e^{(d-D) t}\left|K\left(e^{-t}\right)\right| & \text { for } t \geq 0 \\ 0 & \text { for } t<0\end{cases}
$$

and notice that (2.4) becomes

$$
w(t)=\sum_{t+\log r_{i} \geq 0} r_{i}^{D} w\left(t+\log r_{i}\right)+z(t),
$$

where for $t \geq 0$

$$
z(t)=e^{(d-D) t} R\left(e^{-t}\right)+\sum_{t+\log r_{i}<0} r_{i}^{d} e^{(d-D) t}\left|K\left(r_{i}^{-1} e^{-t}\right)\right|
$$

and $z(t)=0$ for $t<0$. Let $F$ be the probability distribution function that puts mass $r_{i}^{D}$ at the point $-\log r_{i}$. Then (2.5) is equivalent to the renewal equation

$$
w(t)=z(t)+\int_{(0, t]} w(t-s) d F(s) .
$$

Observe that the function $R\left(e^{-t}\right)$ is continuous (see (2.3)) and hence $z$ has only finitely many discontinuities at the points $t=-\log r_{i}$, coming from the second term in (2.6). We also claim the following:

Lemma 2.5. There exist constants $0<C<\infty$ and $\delta>0$ such that

$$
|z(t)| \leq C e^{-t \delta} .
$$

The proof of the lemma is deferred to the next section.

By the lemma and the paragraph preceding it, the function $z$ is directly Riemannintegrable and we may therefore apply the renewal theorem (see [8]). There are two cases to consider.

NON-LATTICE CASE: If the numbers $\log r_{i}$ are not all multiples of some $\lambda>0$, then $F$ is non-lattice and

$$
\lim _{t \rightarrow \infty} w(t)=\frac{\int_{0}^{\infty} z(t) d t}{-\sum_{i=1}^{N} r_{i}^{D} \log r_{i}} .
$$

This shows Theorem 2.3 (i). 
To show (ii) in the non-lattice case observe that, since $K$ is compact, the function $w$ is, by definition, bounded on any finite interval and hence by (2.8)

$$
\lim _{T \rightarrow \infty} \frac{1}{T} \int_{0}^{T} w(t) d t=\frac{\int_{0}^{\infty} z(t) d t}{-\sum_{i=1}^{N} r_{i}^{D} \log r_{i}} .
$$

LATTICE CASE: If there exists some number $\lambda>0$ such that

$$
\left\{\log r_{1}, \ldots, \log r_{N}\right\} \subset \lambda \mathbb{Z}
$$

and $\lambda$ is the largest such number, then $F$ is lattice with span $\lambda$ and so the renewal theorem asserts that for each $s \in[0, \lambda)$

$$
\lim _{n \rightarrow \infty} w(n \lambda+s)=\lambda \frac{\sum_{n=0}^{\infty} z(n \lambda+s)}{-\sum_{i=1}^{N} r_{i}^{D} \log r_{i}} .
$$

We next turn to the proof of Theorem 2.3 (ii) in the lattice case. We begin by observing that $w$ is bounded. This is because $w$ is nonnegative, $\{w(n \lambda)\}_{n \geq 0}$ converges to a finite number, by (2.9) and Lemma 2.5. and, for any $t \geq 0$ and $s \in[0, \lambda)$,

$$
w(t+s)=e^{(d-D)(t+s)}\left|K\left(e^{-t-s}\right)\right| \leq e^{(d-D)(t+s)}\left|K\left(e^{-t}\right)\right| \leq e^{(d-D) \lambda} w(t) .
$$

Next let $L(s)$ denote the limit on the right side of (2.9), for $s \in[0, \lambda)$. By the bounded convergence theorem and (2.9)

$$
\lim _{n \rightarrow \infty} \int_{[0, \lambda)} w(n \lambda+s) d s=\int_{[0, \lambda)} L(s) d s .
$$

Let \lfloor\rfloor denote integer-part and write

$$
\lim _{T \rightarrow \infty} \frac{1}{T} \int_{0}^{T} w(t) d t=\lim _{T \rightarrow \infty} \frac{1}{T} \sum_{n=0}^{\lfloor T / \lambda\rfloor-1} \int_{(0, \lambda]} w(n \lambda+s) d s+\lim _{T \rightarrow \infty} \frac{1}{T} \int_{\lfloor T / \lambda\rfloor \lambda}^{T} w(t) d t .
$$

The first term on the right here converges to $\lambda^{-1} \int_{[0, \lambda)} L(s) d s$, by (2.10) and Cesaro averaging, while the second term tends to 0 by the boundedness of $w$. Hence again

$$
\lim _{T \rightarrow \infty} \frac{1}{T} \int_{0}^{T} w(t) d t=\frac{1}{\lambda} \int_{(0, \lambda]} L(s) d s=\frac{\int_{0}^{\infty} z(t) d t}{-\sum_{i=1}^{N} r_{i}^{D} \log r_{i}} .
$$

This proves (ii) in the lattice case and concludes the proof of the theorem.

2.3. Proof of Lemma 2.5. Recall equation (2.5) defining $z$. The second term on the right is nonnegative, bounded on any neighborhood of 0 and vanishes for $t>\max \left\{-\log r_{1}, \ldots,-\log r_{N}\right\}$. Consequently, it suffices to show that there exist $0<C<\infty$ and $\delta>0$ such that

$$
|R(\epsilon)| \leq C \epsilon^{d-D+\delta}
$$

for all $0<\epsilon<1$. Now recall $(\underline{2.3})$ :

$$
|R(\epsilon)|=\sum_{i=1}^{N}\left|K_{i}(\epsilon)\right|-|K(\epsilon)| \leq \sum_{i \neq j}\left|K_{i}(\epsilon) \cap K_{j}(\epsilon)\right|
$$

and it is therefore enough to show that for some $0<C<\infty$ and $\delta>0$

$$
\left|K_{i}(\epsilon) \cap K_{j}(\epsilon)\right| \leq C \epsilon^{d-D+\delta}
$$

for all $i \neq j$ and $0<\epsilon<1$. 
Towards this end fix $i \neq j$ and let $\epsilon>0$. Call a subset $F$ of $K_{i} \epsilon$-separated if $x, y \in F \Rightarrow \operatorname{dist}(x, y) \geq \epsilon$. Define $Q_{i j}(\epsilon)$ to be the maximum cardinality of an $\epsilon$ separated subset $F$ of $K_{i}$, such that $x \in F \Rightarrow \operatorname{dist}\left(x, K_{j}\right) \leq \epsilon$. Then, by Proposition 3 of reference [12], there exist constants $0<\gamma<\infty$ and $\delta>0$, independent of $\epsilon, i$ and $j$, such that

$$
Q_{i j}(\epsilon) \leq \gamma \epsilon^{\delta-D}
$$

The results of reference [12] require that $\left\{\phi_{1}, \ldots, \phi_{N}\right\}$ satisfies the strong open set condition, i.e., that $K \cap G \neq \emptyset$ for the set $G$ of the open set condition. It is now known however that the strong open set condition is equivalent to the open set condition (Schief [23]).

Now fix $0<\epsilon<1$ and let $F$ be a maximal $2 \epsilon$-separated subset of $K_{i}$, such that for each $z \in F$ we have $\operatorname{dist}\left(z, K_{j}\right) \leq 2 \epsilon$. Then, using the maximality of $F$,

$$
K_{i}(\epsilon) \cap K_{j}(\epsilon) \subseteq \bigcup_{y \in F} B_{3 \epsilon}(y),
$$

where $B_{\rho}(y)$ denotes the ball with center $y$ and radius $\rho$. Since card $F=Q_{i j}(2 \epsilon)$, we have then by (2.12) and (2.13)

$$
\begin{aligned}
\left|K_{i}(\epsilon) \cap K_{j}(\epsilon)\right| & \leq \operatorname{card} F c_{d}(3 \epsilon)^{d} \\
& \leq \gamma c_{d} 3^{d} 2^{\delta-D} \epsilon^{d-D+\delta} \\
& =C \epsilon^{d-D+\delta}
\end{aligned}
$$

where $c_{d}=\frac{[\Gamma(1 / 2)]^{d}}{\Gamma(1+d / 2)}$ is the Lebesgue measure of the unit ball in $\mathbb{R}^{d}$. This shows (2.11) and completes the proof of Lemma 2.5

2.4. Proof of Theorem 2.4. We will actually show that $\epsilon^{(d-D)}|K(\epsilon)|$ is bounded away from 0 , for $0<\epsilon \leq 1$.

Since $\left\{\phi_{1}, \ldots, \phi_{N}\right\}$ satisfies the open set condition, it also satisfies the strong open set condition (cf. [23]); i.e., one can choose the open set $G$ of the open set condition so that $K \cap G \neq \emptyset$. Fix an $x \in K \cap G \neq \emptyset$ and $\delta>0$ such that $B_{\delta}(x) \subset G$.

For $0<\epsilon \leq 1$, define

$$
N(\epsilon)=\left\{\left(i_{1} \ldots i_{n}\right) \in\{1, \ldots, N\}^{*}: r_{i_{1}} \ldots r_{i_{n}}<\epsilon \leq r_{i_{1}} \ldots r_{i_{n-1}}\right\}
$$

First note that since $\sum_{i=1}^{N} r_{i}^{D}=1$ we also have

$$
\sum_{\left(i_{1} \ldots i_{n}\right) \in N(\epsilon)}\left(r_{i_{1}} \ldots r_{i_{n}}\right)^{D}=1
$$

and as each term in the sum is $<\epsilon^{D}$, by the definition of $N(\epsilon)$,

$$
\operatorname{card} N(\epsilon) \geq \epsilon^{-D} \text {. }
$$

We claim that

$$
|K(\epsilon)| \geq c_{d} \epsilon^{d} \operatorname{card} N\left(\epsilon / \delta r_{\min }\right)
$$

where $r_{\min }:=\min r_{i}$. To see this write $x_{i_{1} \ldots i_{n}}=\phi_{i_{1}} \circ \ldots \circ \phi_{i_{n}}(x)$, where $x$ is fixed above, and notice that, since $B_{\delta}(x) \subset G$,

$$
B_{r_{i_{1}} \ldots r_{i_{n}} \delta}\left(x_{i_{1} \ldots i_{n}}\right) \subset \phi_{i_{1}} \circ \ldots \circ \phi_{i_{n}}(G) \text {. }
$$


If $i_{1} \ldots i_{n}$ and $j_{1} \ldots j_{m}$ are two distinct sequences in $N\left(\epsilon / \delta r_{\min }\right)$, then $i_{p} \neq j_{p}$ for some $p \leq \min \{m, n\}$. This implies then that

$$
\phi_{i_{1}} \circ \ldots \circ \phi_{i_{n}}(G) \cap \phi_{j_{1}} \circ \ldots \circ \phi_{j_{m}}(G)=\emptyset
$$

and therefore

$$
B_{r_{i_{1}} \ldots r_{i_{n}} \delta}\left(x_{i_{1} \ldots i_{n}}\right) \cap B_{r_{j_{1}} \ldots r_{j_{m}} \delta}\left(x_{i_{1} \ldots i_{n}}\right)=\emptyset .
$$

But for any sequence $\left(i_{1} \ldots i_{n}\right) \in N\left(\epsilon / \delta r_{\text {min }}\right)$ we have

$$
r_{i_{1}} \ldots r_{i_{n}} \delta \geq \frac{\epsilon}{\delta r_{\min }} r_{i_{n}} \delta \geq \epsilon,
$$

so the balls $B_{\epsilon}\left(x_{i_{1} \ldots i_{n}}\right),\left(i_{1} \ldots i_{n}\right) \in N\left(\epsilon / \delta r_{\min }\right)$, are disjoint. Inequality (2.15) now follows.

Combining (2.14) and (2.15) we have that

$$
\epsilon^{(d-D)}|K(\epsilon)| \geq c_{d} \delta^{D} r_{\min }^{D}>0
$$

for all $0<\epsilon \leq 1$.

\section{Stochastically SELF-Similar Sets}

3.1. Background and statement of main result. In this section we describe the kind of random sets we are considering and state our main result concerning them. The construction below follows Mauldin and Williams ([20]) and can also be found in Falconer $([6])$.

Let $J$ be a nonempty compact set in $\mathbb{R}^{d}$ and write $G:=\operatorname{int}(J)$. We assume that $\bar{G}=J$. Let $\mathcal{S}$ denote the set of all contracting similarities of $\mathbb{R}^{d}$. We assume as given a probability space, on which we have defined a random element $\Phi$, taking either the value e (for empty) or $\Phi=\left\{\phi_{1}, \ldots, \phi_{\nu}\right\}$, where $\nu$ is an integer-valued random variable $(\nu=0$ if $\Phi=\mathrm{e})$ and each $\phi_{i}$ is in $\mathcal{S}$, and furthermore

$$
\begin{aligned}
& \phi_{i}(J) \subseteq J \text { for each } i=1, \ldots, \nu ; \\
& \phi_{i}(G) \cap \phi_{j}(G)=\emptyset \text { whenever } i \neq j, 1 \leq i, j \leq \nu .
\end{aligned}
$$

(See the Appendix for matters of measurability.) We further write $r$ for the constant ratio $|\phi(x)-\phi(y)| /|x-y|, x \neq y$, for a similarity $\phi ; r$ and $\phi$ will always be in this relation in the sequel. Note that $\phi \in \mathcal{S} \Rightarrow r \in(0,1)$.

The random set of interest is defined by means of the family tree of a GaltonWatson branching process. Let $\mathbb{N}^{*}=\{o\} \cup \bigcup_{n=1}^{\infty} \mathbb{N}^{n}$ ( $o$ for 'ancestor') and for each $x \in \mathbb{N}^{*}$ let $\left(\Omega_{x}, \mathcal{F}_{x}, \mathbb{P}_{x}\right)$ be a copy of the above probability space. Set $(\Omega, \mathcal{F}, \mathbb{P})=$ $\prod_{x \in \mathbb{N}^{*}}\left(\Omega_{x}, \mathcal{F}_{x}, \mathbb{P}_{x}\right)$. On $(\Omega, \mathcal{F}, \mathbb{P})$ we then have defined independent and identically distributed random elements $\Phi_{x}=\left\{\phi_{x 1}, \ldots, \phi_{x \nu_{x}}\right\}, x \in \mathbb{N}^{*}\left(\Phi_{x}=\mathrm{e}\right.$ if $\left.\nu_{x}=0\right)$.

Write $\mathcal{G}_{0}=\{o\}$ and inductively define $\mathcal{G}_{n+1}=\emptyset$, if $\mathcal{G}_{n}=\emptyset$, and

$$
\mathcal{G}_{n+1}=\left\{(x, i): x \in \mathcal{G}_{n}, \nu_{x} \neq 0,1 \leq i \leq \nu_{x}\right\},
$$

if $\mathcal{G}_{n} \neq \emptyset$; observe that $\mathcal{G}_{n+1}=\emptyset$ if $\nu_{x}=0$ for all $x \in \mathcal{G}_{n}$. For each $n \geq 0, \mathcal{G}_{n}$ represents the individuals in the $n$-th generation of a Galton-Watson branching process with mean family size $\mathbb{E}[\nu]=\mathbb{E}\left[\operatorname{card} \mathcal{G}_{1}\right]$. Write

$$
\mathcal{P}:=\bigcup_{n=0}^{\infty} \mathcal{G}_{n}
$$




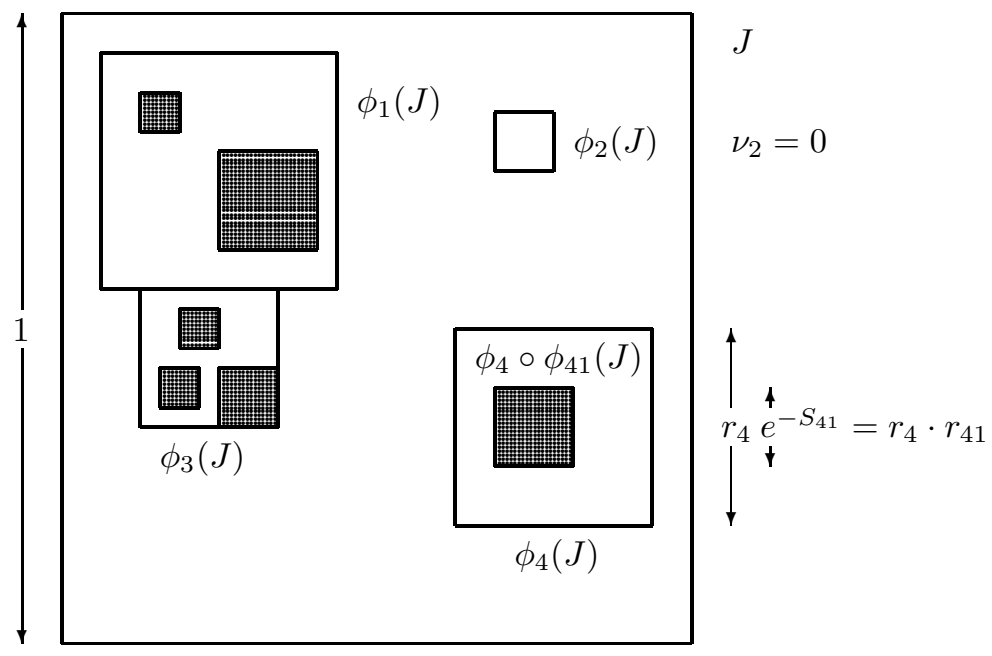

Figure 3. Two steps in the construction of a random set.

for the entire population. Writing $x=x_{1} \ldots x_{n}$ for $x \in \mathcal{G}_{n}$, the set

$$
K:=\bigcap_{n=1}^{\infty} \bigcup_{x \in \mathcal{G}_{n}} \phi_{x_{1}} \circ \phi_{x_{1} x_{2}} \circ \ldots \circ \phi_{x_{1} \ldots x_{n}}(J)
$$

defines then a random compact set in $\mathbb{R}^{d} ; K$ is a stochastically self-similar set.

It is well known (see for example [20]) that $\mathbb{P}(K \neq \emptyset)>0$ iff $\mathbb{E}[\nu]>1$; we will therefore assume throughout that

$$
1<\mathbb{E}[\nu]<\infty .
$$

We now identify a branching random walk naturally associated with the construction, which will be key in the sequel. Set $S_{0}=0$ and for $n \geq 1$ inductively define

$$
S_{x_{1} \ldots x_{n}}=S_{x_{1} \ldots x_{n-1}}+\log r_{x_{1} \ldots x_{n}}^{-1},
$$

where, recall, $r_{x}$ stands for the constant factor of contraction of the similarity $\phi_{x}$. Note that $S_{x}$ is well defined whenever $x \in \mathcal{P}$; for notational convenience set $S_{x}=\infty$ for $x \in \mathbb{N}^{*} \backslash \mathcal{P}$. $\left\{S_{x}: x \in \mathbb{N}^{*}\right\}$ is then a branching random walk with positive step-sizes (since each $\phi_{x}$ is a contraction).

For $\theta \in \mathbb{R}$, set

$$
\begin{gathered}
m(\theta):=\mathbb{E}\left[\sum_{x \in \mathcal{G}_{1}} e^{-\theta S_{x}}\right]=\mathbb{E}\left[\sum_{x \in \mathcal{G}_{1}} r_{x}^{\theta}\right], \\
m^{\prime}(\theta):=\mathbb{E}\left[-\sum_{x \in \mathcal{G}_{1}} S_{x} e^{-\theta S_{x}}\right] .
\end{gathered}
$$

By our assumption (3.1) we have $m(\theta)<\infty$ for all $\theta \geq 0$ and $-m^{\prime}(\theta)<\infty$ for all $\theta>0$. The function $m$ is a decreasing convex $C^{\infty}$ function on $(0, \infty)$, which is continuous on $[0, \infty)$, with $m(0)=\mathbb{E}[\nu]>1$ and $m(\infty)=0$. Consequently, there exists a unique number $D>0$ such that $m(D)=1$. By a well known result, obtained independently by Mauldin and Williams ([20]) and Falconer ([6]), the 
number $D$ is then the Hausdorff dimension of the random set $K$, almost surely on the event $\{K \neq \emptyset\}$. That it is also the Minkowski dimension of $K$ (on $\{K \neq \emptyset\}$ ) follows easily from the results of the present paper and is a well known fact.

We now state the main result of this paper for stochastically self-similar sets. Observe that the random set $K$ is, probabilistically, uniquely determined by the compact set $J$ and the random set of similarities $\Phi=\left\{\phi_{1}, \ldots, \phi_{\nu}\right\}$. Also recall that $G=\operatorname{int}(J)$, that $F(\epsilon)$ denotes the $\epsilon$-neighborhood of a compact set $F$ (Notation 2.1) and that || denotes $d$-dimensional Lebesgue measure.

Theorem 3.1. Assume of $\Phi$ and $J$ that $1<\mathbb{E}[\nu]<\infty$ and that

$$
\mathbb{P}(K \cap G \neq \emptyset)>0 .
$$

(i) Assume that the measure $A \mapsto \mathbb{E}\left[\sum_{x \in \mathcal{G}_{1}} \mathbf{1}_{A}\left(S_{x}\right)\right]$ is non-lattice; i.e., it does not concentrate on an additive subgroup of $\mathbb{R}$ of the form $\lambda \mathbb{Z}$ for some $\lambda>0$. Then the following limit exists and is finite a.s.:

$$
L:=\lim _{\epsilon \rightarrow 0} \frac{|K(\epsilon)|}{\epsilon^{d-D}} .
$$

(ii) The following limit always exists and is finite a.s.:

$$
L:=\lim _{T \rightarrow \infty} \frac{1}{T} \int_{0}^{T} e^{(d-D) t}\left|K\left(e^{-t}\right)\right| d t .
$$

Note. As in the deterministic case, the random variable $L$ is always defined by part (ii) of the above theorem. When the limit in (i) exists, it necessarily equals the limit in (ii); hence there is no ambiguity in using the same letter for both limits.

Theorem 3.2. Set $M_{1}=\sum_{x \in \mathcal{G}_{1}} r_{x}^{D}$.

(i) If $\mathbb{E}\left[M_{1} \log ^{+} M_{1}\right]=\infty$, then $L=0$ with probability 1 .

(ii) If $\mathbb{E}\left[M_{1} \log ^{+} M_{1}\right]<\infty$, then $\mathbb{P}(L>0 \mid K \neq \emptyset)=1$.

Remark. Note that $\mathbb{E}\left[\nu \log ^{+} \nu\right]<\infty$ implies $\mathbb{E}\left[M_{1} \log ^{+} M_{1}\right]<\infty$.

We defer the proof of Theorem 3.1 to section 3.3 and that of Theorem 3.2 to section 3.5.

3.2. Renewal theorem for branching random walks. Let $\left\{S_{x}: x \in \mathbb{N}^{*}\right\}$ be the branching random walk associated with the construction of $K$ and recall that this walk has positive steps. Denote by $\xi$ the random measure (point process) $\xi(A):=\sum_{x \in \mathcal{G}_{1}} \mathbf{1}_{A}\left(S_{x}\right)$, for Borel sets $A$ in $\mathbb{R}$. Since the walk has positive steps, $\xi$ is concentrated on $(0, \infty)$.

Recall that $D$ is the unique solution to the equation $\mathbb{E}\left[\sum_{x \in \mathcal{G}_{1}} e^{-D S_{x}}\right]=1$ and set

$$
M_{n}:=\sum_{x \in \mathcal{G}_{n}} e^{-D S_{x}}, \quad n \geq 0 .
$$

A straightforward computation shows that the sequence $\left(M_{n}\right)_{n \geq 0}$ is a non-negative martingale (relative to the natural filtration associated with the successive steps of the construction of $K$ ). Denote

$$
M_{\infty}:=\lim _{n \rightarrow \infty} M_{n} .
$$

The following theorem is due to Biggins ([3]); see Lyons [17] for a conceptual proof, following work by Lyons, Pemantle and Peres [18]. 
Theorem 3.3 (Biggins). The following are equivalent:

(i) $\mathbb{E}\left[M_{1} \log ^{+} M_{1}\right]<\infty$;

(ii) $\mathbb{P}\left(M_{\infty}=0\right)<1$;

(iii) $\mathbb{P}\left(M_{\infty}>0 \mid\right.$ non-extinction $)=1$;

(iv) $\mathbb{E}\left[M_{\infty}\right]=1$;

(v) $M_{n} \longrightarrow M_{\infty}$ in $L^{1}$.

Recall that our underlying probability space $(\Omega, \mathcal{F}, \mathbb{P})=\prod_{x \in \mathbb{N}^{*}}\left(\Omega_{x}, \mathcal{F}_{x}, \mathbb{P}_{x}\right)$ is a product-space. For each $x \in \mathbb{N}^{*}$ let $\sigma_{x}: \Omega \rightarrow \Omega$ be the shift, defined by

$$
\left(\sigma_{x} \omega\right)_{y}=\omega_{x y}
$$

where, if $x=x_{1} \ldots x_{n}$ and $y=y_{1} \ldots y_{m}$, then $x y=x_{1} \ldots x_{n} y_{1} \ldots y_{m}$.

Let $Z=\left\{Z_{t}: t \in \mathbb{R}\right\}$ be a stochastic process on $(\Omega, \mathcal{F}, \mathbb{P})$, which is jointly measurable in $(\omega, t)$ and is separable and takes values in the space of functions which possess both left- and right-hand limits and vanish on $(-\infty, 0)$. Given such a process $Z$, write $Z^{x}=\left\{Z_{t}^{x}: t \in \mathbb{R}\right\}$ for the process

$$
Z_{t}^{x}(\omega)=Z_{t}\left(\sigma_{x} \omega\right)
$$

for $x \in \mathbb{N}^{*}$. So if $Z$ is some process associated with the branching random walk $\left\{S_{x}: x \in \mathbb{N}^{*}\right\}$ (or the construction leading to $K$ ), then $Z^{x}$ is the corresponding process associated with that part of the population which emanates from individual $x$. The following theorem is due to Nerman 22 and is an analogue of the renewal theorem for ordinary random walks.

Theorem 3.4. Suppose that there exists a non-increasing and integrable function $h:[0, \infty) \rightarrow(0, \infty)$, such that

$$
\mathbb{E}\left[\sup _{t \geq 0} \frac{e^{-D t}\left|Z_{t}\right|}{h(t)}\right]<\infty .
$$

(i) [Nerman] If the measure $A \mapsto \mathbb{E}[\xi(A)]$ is non-lattice, then a.s., as $t \longrightarrow \infty$,

$$
e^{-D t} \sum_{x \in \mathcal{P}} Z_{t-S_{x}}^{x} \longrightarrow \frac{M_{\infty}}{-m^{\prime}(D)} \int_{0}^{\infty} e^{-D s} \mathbb{E}\left[Z_{s}\right] d s .
$$

(ii) If the measure $A \mapsto \mathbb{E}[\xi(A)]$ is lattice, with span $\lambda>0$, then for every $s \in$ $[0, \lambda)$, we have that a.s., as $n \longrightarrow \infty$,

$$
e^{-D n \lambda} \sum_{x \in \mathcal{P}} Z_{s+n \lambda-S_{x}}^{x} \longrightarrow \frac{\lambda M_{\infty}}{-m^{\prime}(D)} \sum_{n=0}^{\infty} e^{-D n \lambda} \mathbb{E}\left[Z_{n \lambda+s}\right] .
$$

Remark. Recall our standing assumption $1<\mathbb{E}[\nu]<\infty$. Nerman's theorem actually requires less than $\mathbb{E}[\nu]<\infty$; see [22] for the precise conditions needed. Furthermore, the lattice case does not require $Z$ to have sample paths which possess right- and left-hand limits.

Proof. Assertion (i) is Theorem 5.4 of Nerman [22].

(ii) A close examination of Nerman's proof reveals that the non-lattice assumption is only used in two places: on page 384, Proof of Theorem 5.4 from Corollary 5.11 and in the second part of the Proof of Lemma 5.10 (establishing (5.53)). Of these the first is irrelevant for the lattice case and his proof of Lemma 5.10 (with obvious modifications) yields the following statement for the lattice case: If $p \in \mathbb{N}$ is 
such that $\mathbb{E}[\xi(0, p \lambda]]>1$ and (3.4) is satisfied (and given our standing assumption $1<\mathbb{E}[\nu]<\infty)$, then, for any $t \geq 0$,

$$
e^{-D k p \lambda} \sum_{x \in \mathcal{P}} Z_{t+k p \lambda-S_{x}}^{x} \longrightarrow \frac{\lambda M_{\infty}}{-m^{\prime}(D)} \sum_{n=-\infty}^{\infty} e^{-D n \lambda} \mathbb{E}\left[Z_{t+n \lambda}\right]
$$

as $k \longrightarrow \infty$, on a set $B_{t}$ with $\mathbb{P}\left(B_{t}\right)=1$.

Fix $s \in[0, \lambda)$ and set $A_{s}=B_{s} \cap B_{s+\lambda} \cap \ldots \cap B_{s+(p-1) \lambda}$. Then $\mathbb{P}\left(A_{s}\right)=1$. On $A_{s}$, and given $\epsilon>0$, choose $k_{\epsilon}$ so that, for $k \geq k_{\epsilon}, e^{-D k p \lambda} \sum_{x \in \mathcal{P}} Z_{t+k p \lambda-S_{x}}^{x}$ is within $\pm \epsilon$ from its limit for all $t \in\{s, s+\lambda, \ldots, s+(p-1) \lambda\}$. For an arbitrary $n \in \mathbb{N}$ write $n=k(n) p+q, 0 \leq q<p$. Then, if $n \geq k_{\epsilon} p+p-1$,

$$
e^{-D n \lambda} \sum_{x \in \mathcal{P}} Z_{s+n \lambda-S_{x}}^{x}=e^{-D q \lambda} e^{-D k(n) p \lambda} \sum_{x \in \mathcal{P}} Z_{s+q \lambda+k(n) p \lambda-S_{x}}^{x}
$$

is within $\pm \epsilon$ from its limit

$$
e^{-D q \lambda} \frac{\lambda M_{\infty}}{-m^{\prime}(D)} \sum_{m=-\infty}^{\infty} e^{-D m \lambda} \mathbb{E}\left[Z_{s+q \lambda+m \lambda}\right]=\frac{\lambda M_{\infty}}{-m^{\prime}(D)} \sum_{m=-\infty}^{\infty} e^{-D m \lambda} \mathbb{E}\left[Z_{s+m \lambda}\right] .
$$

This proves the convergence asserted in (ii).

We close this section with a simple but useful fact. The sequence

$$
M_{n}^{(\theta)}:=[m(\theta)]^{-n} \sum_{x \in \mathcal{G}_{n}} e^{-\theta S_{x}}, \quad n \geq 0,
$$

is a nonnegative martingale, for each $\theta \geq 0$, as then $m(\theta)<\infty$. Hence $M_{n}^{(\theta)}$ converges to a finite limit $M_{\infty}^{(\theta)}$. For $\theta>D$ we have $0<m(\theta)<1$ and therefore $\sum_{x \in \mathcal{G}_{n}} e^{-\theta S_{x}} \longrightarrow 0$, as $n \longrightarrow \infty$. Consequently, with probability equal to one,

$$
\min _{x \in \mathcal{G}_{n}} S_{x} \longrightarrow \infty, \quad \text { as } n \longrightarrow \infty .
$$

3.3. Proof of Theorem 3.1. Recall that $|K(\epsilon)|$ denotes the $\epsilon$-neighborhood of the random set $K$. Then

$$
|K(\epsilon)|=\sum_{x \in \mathcal{G}_{1}}\left|\left[K \cap \phi_{x}(J)\right](\epsilon)\right|-Q(\epsilon) .
$$

Let $K_{x}=K \cap \phi_{x}(J)$ and $K^{x}=\phi_{x}^{-1}\left(K \cap \phi_{x}(J)\right)$; observe that the set $K^{x}$ has the same distribution as $K$ and that the $K^{x}, x \in \mathcal{G}_{1}$, are independent (given the $\sigma$-algebra $\left.\sigma\left(\Phi_{0}\right)\right)$. Since each $\phi_{x}$ is a similarity,

$$
\left|K_{x}(\epsilon)\right|=e^{-d S_{x}}\left|K^{x}\left(\epsilon e^{S_{x}}\right)\right|
$$

and so, with

$$
R_{t}=Q\left(e^{-t}\right) \mathbf{1}_{[0, \infty)}(t)
$$

and

$$
W_{t}=e^{(d-D) t}\left|K\left(e^{-t}\right)\right| \mathbf{1}_{[0, \infty)}(t),
$$

we have that

$$
W_{t}=\sum_{x \in \mathcal{G}_{1}} e^{-D S_{x}} W_{t-S_{x}}^{x}+e^{-D t} Z_{t},
$$


where, for $t \geq 0$,

$$
Z_{t}:=\sum_{x \in \mathcal{G}_{1}} e^{d\left(t-S_{x}\right)}\left|K^{x}\left(e^{-t+S_{x}}\right)\right| \mathbf{1}_{(t, \infty)}\left(S_{x}\right)-e^{d t} R_{t}
$$

and $Z_{t}:=0$ for $t<0$. Then, upon iterating (3.8), we obtain

$$
W_{t}=e^{-D t} \sum_{x \in \mathcal{P}} Z_{t-S_{x}}^{x} .
$$

Observe that the process $Z=\left\{Z_{t}: t \in \mathbb{R}\right\}$ satisfies $Z_{t}=0$ for $t<0$ and has paths which are left-continuous with right-hand limits; as a matter of fact $Z$ is continuous except possibly at the points $S_{x}, x \in \mathcal{G}_{1}$. We shall apply Theorem 3.4 and to do so we need to verify that condition (3.4) holds. We will do this in two separate lemmata whose proofs we postpone to the end of this section.

Lemma 3.5. There exists a non-increasing and integrable function $g:[0, \infty) \rightarrow$ $(0, \infty)$, for which

$$
\mathbb{E}\left[\sup _{t \geq 0} \frac{e^{-D t}}{g(t)} \sum_{x \in \mathcal{G}_{1}} e^{d\left(t-S_{x}\right)}\left|K^{x}\left(e^{-t+S_{x}}\right)\right| \mathbf{1}_{(t, \infty)}\left(S_{x}\right)\right]<\infty .
$$

Lemma 3.6. There exists a non-increasing and integrable function $h:[0, \infty) \rightarrow$ $(0, \infty)$, for which

$$
\mathbb{E}\left[\sup _{t \geq 0} \frac{e^{(d-D) t} R_{t}}{h(t)}\right]<\infty .
$$

It follows from (3.9) and the two lemmata that

$$
\mathbb{E}\left[\sup _{t \geq 0} \frac{e^{-D t}\left|Z_{t}\right|}{h(t)+g(t)}\right]<\infty,
$$

with $h+g$ non-increasing and integrable.

In view of (3.11) and the paragraph preceding Lemma 3.5. we may now apply Theorem 3.4 to 3.10 .

(i) Assume that the measure $A \mapsto \mathbb{E}[\xi(A)]$ is non-lattice. Then by Theorem 3.4 we have that with probability equal to one, as $t \longrightarrow \infty$,

$$
W_{t} \longrightarrow \frac{M_{\infty}}{-m^{\prime}(D)} \int_{0}^{\infty} e^{-D s} \mathbb{E}\left[Z_{s}\right] d s .
$$

This shows Theorem 3.1 (i).

(ii) Observe that, because $T \mapsto T^{-1} \int_{0}^{T} W_{t}(\omega) d t$ is continuous, for each $\omega$, the functions

$$
\omega \mapsto \limsup _{T \rightarrow \infty} \frac{1}{T} \int_{0}^{T} W_{t}(\omega) d t \quad \text { and } \quad \omega \mapsto \liminf _{T \rightarrow \infty} \frac{1}{T} \int_{0}^{T} W_{t}(\omega) d t
$$

are measurable and therefore the set of $\omega$ for which $\lim _{T \rightarrow \infty} T^{-1} \int_{0}^{T} W_{t}(\omega) d t$ exists is measurable. It therefore suffices to show that this set of convergence contains a set of probability one.

Assume first $A \mapsto \mathbb{E}[\xi(A)]$ is non-lattice. From (i) we have that

$$
W_{t}=e^{(d-D) t}\left|K\left(e^{-t}\right)\right| \longrightarrow L, \quad \text { a.s. }
$$


as $t \longrightarrow \infty$. As $W_{t}(\omega)$ is bounded on finite intervals (actually uniformly in $\omega$, by compactness of $J$ ), it follows that, as $T \longrightarrow \infty$,

$$
\frac{1}{T} \int_{0}^{T} W_{t} d t \longrightarrow L
$$

on the event $\left\{\omega: W_{t}(\omega) \longrightarrow L(\omega)\right\}$, i.e., on a set of probability one.

We next turn to the lattice case. Assume that the measure $A \mapsto \mathbb{E}[\xi(A)]$ is concentrated on a lattice $\lambda \mathbb{Z}$ and that $\lambda$ is the largest such number.

By Theorem 3.4 (ii), for each $s \in[0, \lambda)$, there exists a set $A_{s}$ of probability one, such that, on $A_{s}$, as $n \longrightarrow \infty$,

$$
W_{s+n \lambda} \longrightarrow \lambda e^{-D s} \frac{M_{\infty}}{-m^{\prime}(D)} \sum_{k=0}^{\infty} e^{-D k \lambda} \mathbb{E}\left[Z_{s+k \lambda}\right]
$$

Call the limit on the right-hand side $L_{s}$ and set $A:=\bigcap_{r} A_{r}$, the intersection being over all rational $r$ in $[0, \lambda)$. Then $\mathbb{P}(A)=1$ and

$$
\lim _{n \longrightarrow \infty} W_{r+n \lambda}(\omega)=L_{r}(\omega), \quad \text { for all } \omega \in A \text { and } r \in \mathbb{Q} \cap[0, \lambda) .
$$

For given $s \in[0, \lambda)$ have sequences $\left\{r_{n}\right\}$ and $\left\{q_{n}\right\}$ in $\mathbb{Q} \cap[0, \lambda)$ such that $r_{n} \uparrow s$ and $q_{n} \downarrow s$. Since for $t, s \geq 0$ one has $K\left(e^{-t-s}\right) \subseteq K\left(e^{-t}\right)$, it follows that

$$
W_{t+s} \leq e^{(d-D) s} W_{t} \quad(t, s \geq 0)
$$

Using this and (3.13) we obtain that, for all $\omega \in A$ and all $k \geq 1$,

$$
\begin{aligned}
e^{-(d-D)\left(q_{k}-s\right)} L_{q_{k}}(\omega) & \leq \liminf _{n \rightarrow \infty} W_{s+n \lambda}(\omega) \\
& \leq \limsup _{n \rightarrow \infty} W_{s+n \lambda}(\omega) \leq e^{(d-D)\left(s-r_{k}\right)} L_{r_{k}}(\omega) .
\end{aligned}
$$

Next observe that the function $t \mapsto \mathbb{E}\left[Z_{t}\right]$ has left- and right-hand limits. This is so because the sample-paths of $Z$ have this property and we can use the dominated convergence theorem by (3.11). Consequently, the set $C$ where this function is continuous has full Lebesgue measure; as a matter of fact $C^{c}$ is countable. Using (3.11) again, we conclude that the function

$$
t \mapsto \sum_{k=0}^{\infty} e^{-D k \lambda} \mathbb{E}\left[Z_{t+k \lambda}\right], \quad t \in[0, \lambda),
$$

is continuous outside $\bigcup_{k=0}^{\infty}\left(C^{c}-k \lambda\right)$, a countable set. Thus for Lebesgue-a.a. $s \in[0, \lambda)$ we have that $L_{q_{k}}(\omega) \longrightarrow L_{s}(\omega)$ and $L_{r_{k}}(\omega) \longrightarrow L_{s}(\omega)$, as $k \longrightarrow \infty$ (recall the definition of $\left.L_{s}\right)$, and so by (3.14),

$$
W_{s+n \lambda}(\omega) \longrightarrow L_{s}(\omega) \quad(n \longrightarrow \infty)
$$

for all $\omega \in A$. Just as in the deterministic case now, we can conclude that

$$
\frac{1}{T} \int_{0}^{T} W_{t}(\omega) d t \longrightarrow \frac{1}{\lambda} \int_{[0, \lambda)} L_{s}(\omega) d s=\frac{M_{\infty}(\omega)}{-m^{\prime}(D)} \int_{0}^{\infty} e^{-D s} \mathbb{E}\left[Z_{s}\right] d s,
$$

as $T \longrightarrow \infty$, for all $\omega \in A$, i.e., on a set of probability one. This proves Theorem 3.1 (ii) in the lattice case and completes the proof of the theorem.

Proof of Lemma 3.5. Recall that $c_{d}$ denotes the Lebesgue measure of the unit ball in $\mathbb{R}^{d}$ and observe that, for any $x \in \mathbb{N}^{*}$,

$$
\left|K^{x}(\epsilon)\right| \leq|J(\epsilon)| \leq c_{d}[\operatorname{diam}(J)+\epsilon]^{d} .
$$


Hence, if $S_{x}>t$,

$$
e^{d\left(t-S_{x}\right)}\left|K^{x}\left(e^{-t+S_{x}}\right)\right| \leq c_{d} \sup _{\epsilon>1}\left(\frac{\operatorname{diam}(J)+\epsilon}{\epsilon}\right)^{d}=c_{d}[\operatorname{diam}(J)+1]^{d} .
$$

It follows that, for $g(t)=e^{-D t} \mathbf{1}_{[0, \infty)}(t)$,

$$
\begin{aligned}
\mathbb{E}\left[\sup _{t \geq 0} \frac{e^{-D t}}{g(t)} \sum_{x \in \mathcal{G}_{1}} e^{d\left(t-S_{x}\right)}\left|K^{x}\left(e^{-t+S_{x}}\right)\right| \mathbf{1}_{(t, \infty)}\left(S_{x}\right)\right] \\
\leq c_{d}[\operatorname{diam}(J)+1]^{d} \mathbb{E}[\nu]<\infty .
\end{aligned}
$$

Proof of Lemma [3.6. The proof is a probabilistic analogue of the proof of the key estimate of reference [12. It is rather long and we break it up into several steps.

Assume (3.2), that is,

$$
\mathbb{P}(K \cap G \neq \emptyset)>0
$$

For $\alpha>0$ and $p \in \mathbb{N}$ let

$$
\mathcal{G}_{p}(\alpha)=\left\{x \in \mathcal{G}_{p}: \exists v \in \phi_{x_{1}} \circ \ldots \circ \phi_{x_{1} \ldots x_{p}}(J) \text { with } \operatorname{dist}\left(v, G^{c}\right) \leq \alpha\right\} .
$$

Claim 1. There exist $p \in \mathbb{N}$ and $\alpha>0$ such that $\mathbb{P}\left(\mathcal{G}_{p} \backslash \mathcal{G}_{p}(\alpha) \neq 0\right)>0$.

Proof. Let $\partial \mathcal{P}$ consist of all infinite sequences $x=x_{1} x_{2} \ldots \in \mathbb{N}^{\mathbb{N}}$ for which

$$
x_{1} \ldots x_{n} \in \mathcal{G}_{n} \quad \text { for all } n \geq 1 .
$$

By (3.6) and nestedness, we have that $\bigcap_{n \geq 1} \phi_{x_{1}} \circ \ldots \circ \phi_{x_{1} \ldots x_{n}}(J)$ is a singleton for each $x \in \partial \mathcal{P}$, whose unique element we denote by $v_{x}$; so $K=\left\{v_{x}: x \in \partial \mathcal{P}\right\}$.

Suppose now that $\mathbb{P}\left(\mathcal{G}_{p}=\mathcal{G}_{p}(\alpha)\right)=1$, for all $p \in \mathbb{N}$ and $\alpha>0$. Then $\Omega^{\prime}=$ $\bigcap_{\alpha \in \mathbb{Q}^{+}} \bigcap_{p \geq 1}\left\{\mathcal{G}_{p}=\mathcal{G}_{p}(\alpha)\right\}$ has probability one. Now either $K=\emptyset$ or if $v \in K$, then $v=v_{x}$ for some $x \in \partial \mathcal{P}$; then $v_{x} \in \phi_{x_{1}} \circ \ldots \circ \phi_{x_{1} \ldots x_{n}}(J)$ for all $n \geq 1$. On $\Omega^{\prime}$ we have $x_{1} \ldots x_{n} \in \mathcal{G}_{n}(\alpha)$ for all $n \geq 1$ and all $\alpha \in \mathbb{Q}^{+}$and so

$$
\operatorname{dist}\left(v_{x}, G^{c}\right) \leq e^{-S_{x_{1} \ldots x_{n}}} \operatorname{diam}(J)+\alpha,
$$

which, together with (3.6) again, implies that $v_{x} \in G^{c}$. This shows that $K \cap G=\emptyset$ on $\Omega^{\prime}$, creating a contradiction.

From now on we fix a $p$ and an $\alpha$ as in Claim 1. Let

$$
\tilde{\mathcal{G}}_{0}=\mathcal{G}_{0}, \ldots, \tilde{\mathcal{G}}_{p-1}=\mathcal{G}_{p-1}, \tilde{\mathcal{G}}_{p}=\mathcal{G}_{p}(\alpha)
$$

and for $n>p$ let $\tilde{\mathcal{G}}_{n}$ consist of those $x=x_{1} \ldots x_{n} \in \mathcal{G}_{n}$ which have the following property: for each $1 \leq i \leq n-p+1$ there exists $v \in \phi_{x_{1} \ldots x_{i}} \circ \ldots \circ \phi_{x_{1} \ldots x_{i+p-1}}(J)$ with $\operatorname{dist}\left(v, G^{c}\right) \leq \alpha$. Set also $\tilde{\mathcal{P}}=\bigcup_{n \geq 0} \tilde{\mathcal{G}}_{n}$.

For $t \geq 0$ let $\mathcal{P}_{t}$ consist of those $x=x_{1} \ldots x_{m} \in \mathcal{P}$ for which

$$
S_{x_{1} \ldots x_{m-1}} \leq t<S_{x_{1} \ldots x_{m}}
$$

for $t<0$ let $\mathcal{P}_{t}=\{o\}$ and define $\tilde{\mathcal{P}}_{t}$ accordingly.

For $\epsilon>0$, let $Q(\epsilon)$ be the maximum cardinality of an $\epsilon$-separated subset $F$ of $K$, such that $\operatorname{dist}\left(v, G^{c}\right) \leq \epsilon$ for all $v \in F$. Finally, set $s:=\log \alpha^{-1}+\log (\operatorname{diam}(J))$.

Claim 2. With $t=\log \epsilon^{-1}+\log (\operatorname{diam}(J))$, we have that for all $0<\epsilon \leq \alpha$

$$
Q(\epsilon) \leq \sum_{x \in \tilde{\mathcal{P}}_{t-s}} \operatorname{card} \mathcal{P}_{t-S_{x}}^{x}
$$


Proof. Let $F$ be an $\epsilon$-separated subset of $K$, such that $\operatorname{dist}\left(v, G^{c}\right) \leq \epsilon$ for all $v \in F$. By the representation $K=\left\{v_{x}: x \in \partial \mathcal{P}\right\}$ (see the proof of Claim 1), to each point $v \in F$ corresponds an element $x=x_{1} \ldots x_{m} \in \mathcal{P}_{t}$, and different elements of $F$ have different representatives in $\mathcal{P}_{t}$, because $F$ is $\epsilon$-separated. Now given $x=x_{1} \ldots x_{m} \in \mathcal{P}_{t}$ let $q=q_{x}$ be the unique integer in $\{1, \ldots, m\}$ for which

$$
S_{x_{1} \ldots x_{q-1}} \leq \log \left(\frac{\alpha}{\epsilon}\right)<S_{x_{1} \ldots x_{q}} .
$$

We claim that if $x=x_{1} \ldots x_{m} \in \mathcal{P}_{t}$ corresponds to an element $v_{x} \in F$, then $x_{1} \ldots x_{q} \in \tilde{\mathcal{G}}_{q}$. For if not, then for some $1 \leq i \leq q-p+1$ we would have that $\operatorname{dist}\left(u, G^{c}\right)>\alpha$, for all $u \in \phi_{x_{1} \ldots x_{i}} \circ \ldots \circ \phi_{x_{1} \ldots x_{i+p-1}}(J)$, so

$$
\begin{aligned}
\operatorname{dist} & \left(\phi_{x_{1}} \circ \ldots \circ \phi_{x_{1} \ldots x_{i+p-1}}(u), \phi_{x_{1}} \circ \ldots \circ \phi_{x_{1} \ldots x_{i-1}}\left(G^{c}\right)\right) \\
& =\exp \left(-S_{x_{1} \ldots x_{i-1}}\right) \operatorname{dist}\left(\phi_{x_{1} \ldots x_{i}} \circ \ldots \circ \phi_{x_{1} \ldots x_{i+p-1}}(u), G^{c}\right) \\
& >\exp \left(-S_{x_{1} \ldots x_{i-1}}\right) \alpha \\
& \geq \exp \left(-S_{x_{1} \ldots x_{q-1}}\right) \alpha \geq \epsilon,
\end{aligned}
$$

for all $u \in J$; equivalently,

$$
\operatorname{dist}\left(u, \phi_{x_{1}} \circ \ldots \circ \phi_{x_{1} \ldots x_{i-1}}\left(G^{c}\right)\right)>\epsilon,
$$

for all $u \in \phi_{x_{1}} \circ \ldots \circ \phi_{x_{1} \ldots x_{i+p-1}}(J)$ and so in particular (3.17) would be true for $u=$ $v_{x}$. But as $G^{c} \subseteq \phi_{x_{1}} \circ \ldots \circ \phi_{x_{1} \ldots x_{i-1}}\left(G^{c}\right)$ we then would have that $\operatorname{dist}\left(v_{x}, G^{c}\right)>\epsilon$, contradicting the fact that $v_{x} \in F$.

It now follows from the above that for $0<\epsilon \leq \alpha$

$$
Q(\epsilon) \leq \sum_{x \in \mathcal{P}_{t}} \mathbf{1}_{\tilde{\mathcal{G}}_{q}}\left(x_{1} \ldots x_{q}\right)=\sum_{x \in \tilde{\mathcal{P}}_{t-s}} \operatorname{card} \mathcal{P}_{t-S_{x}}^{x} .
$$

Now let $\tilde{D}$ be the unique solution to

$$
\mathbb{E}\left[\sum_{x \in \tilde{\mathcal{G}}_{p}} e^{-\tilde{D} S_{x}}\right]=1
$$

because $\mathbb{P}\left(\mathcal{G}_{p} \backslash \tilde{\mathcal{G}}_{p} \neq \emptyset\right)>0$, by our choice of $p$ and $\alpha$, we must have $\tilde{D}<D$.

Claim 3. For any $\delta>0$ we have

$$
\mathbb{E}\left[\sup _{0<\epsilon \leq \alpha} \epsilon^{\tilde{D}+\delta} Q(\epsilon)\right]<\infty .
$$

Proof. Assume first that $p=1$. Then $\left\{\tilde{\mathcal{G}}_{n}: n \geq 0\right\}$ corresponds to a Galton-Watson branching process, just like $\left\{\mathcal{G}_{n}: n \geq 0\right\}$, and $\left\{S_{x}: x \in \tilde{\mathcal{P}}\right\}$ is a branching random walk, just like $\left\{S_{x}: x \in \mathcal{P}\right\}$, with different parameters however.

Recall the random measure $\xi(A)=\sum_{x \in \mathcal{G}_{1}} \mathbf{1}_{A}\left(S_{x}\right)$; then, for any $\theta \geq 0$ and $t \geq 0$,

$$
\operatorname{card} \mathcal{P}_{t}=\sum_{n=0}^{\infty} \sum_{x \in \mathcal{G}_{n}} \mathbf{1}_{[0, t]}\left(S_{x}\right) \xi^{x}\left(t-S_{x}, \infty\right) \leq e^{\theta t} \sum_{x \in \mathcal{P}} e^{-\theta S_{x}} \nu_{x} .
$$

Set $V_{\theta}=\sum_{x \in \mathcal{P}} e^{-\theta S_{x}} \nu_{x}$ and recall that $\mathcal{P}_{t}=\{o\}$ for $t<0$. By (3.19) then

$$
\sum_{x \in \tilde{\mathcal{P}}_{t-s}} \operatorname{card} \mathcal{P}_{t-S_{x}}^{x} \leq \sum_{x \in \tilde{\mathcal{P}}_{t-s}}\left[e^{\theta\left(t-S_{x}\right)} V_{\theta}^{x} \mathbf{1}_{[0, t]}\left(S_{x}\right)+\mathbf{1}_{(t, \infty)}\left(S_{x}\right)\right] .
$$


Since $x \in \tilde{\mathcal{P}}_{t-s} \Rightarrow S_{x}>t-s$, we then have that

$$
\sum_{x \in \tilde{\mathcal{P}}_{t-s}} \operatorname{card} \mathcal{P}_{t-S_{x}}^{x} \leq e^{\theta s} \sum_{x \in \tilde{\mathcal{P}}_{t-s}} V_{\theta}^{x} \mathbf{1}_{[0, t]}\left(S_{x}\right)+\operatorname{card} \tilde{\mathcal{P}}_{t-s}
$$

Now assume $t \geq s$ and decompose $\tilde{\mathcal{P}}_{t-s}$ as in (3.19) to obtain

$$
\begin{aligned}
\sum_{x \in \tilde{\mathcal{P}}_{t-s}} V_{\theta}^{x} \mathbf{1}_{[0, t]}\left(S_{x}\right) & =\sum_{n=0}^{\infty} \sum_{x \in \tilde{\mathcal{G}}_{n}} \mathbf{1}_{[0, t-s]}\left(S_{x}\right) \sum_{y \in \tilde{\mathcal{G}}_{1}^{x}} \mathbf{1}_{(t-s, t]}\left(S_{x y}\right) V_{\theta}^{x y} \\
& \leq e^{\zeta(t-s)} \sum_{x \in \tilde{\mathcal{P}}} e^{-\zeta S_{x}} \sum_{y \in \tilde{\mathcal{G}}_{1}^{x}} V_{\theta}^{x y}
\end{aligned}
$$

and

$$
\operatorname{card} \tilde{\mathcal{P}}_{t-s} \leq e^{\zeta(t-s)} \sum_{x \in \tilde{\mathcal{P}}} e^{-\zeta S_{x}} \tilde{\nu}_{x},
$$

for any $\zeta \geq 0$. Combining (3.20), 3.21) and (3.22) we finally have that

$$
\sup _{t \geq s}\left\{e^{-\zeta t} \sum_{x \in \tilde{\mathcal{P}}_{t-s}} \operatorname{card} \mathcal{P}_{t-S_{x}}^{x}\right\} \leq e^{(\theta-\zeta) s} \sum_{x \in \tilde{\mathcal{P}}} e^{-\zeta S_{x}} \sum_{y \in \tilde{\mathcal{G}}_{1}^{x}}\left(V_{\theta}^{x y}+1\right)
$$

for all $\theta, \zeta \geq 0$. We will now show that the right-hand side of (3.23) has finite expectation for the choice $\zeta=\tilde{D}+\delta$ and $\theta=D+\delta$. This, in conjunction with Claim 2, will prove Claim 3 in the case $p=1$.

Let $\mathcal{F}_{n}=\sigma\left(\Phi_{x}:|x|<n\right)$, for $n \geq 1$, where here $|x|$ denotes the length of the finite sequence $x$. Write

$$
\mathbb{E}\left[\sum_{x \in \tilde{\mathcal{P}}} e^{-\zeta S_{x}} \sum_{y \in \tilde{\mathcal{G}}_{1}^{x}}\left(V_{\theta}^{x y}+1\right)\right]=\sum_{n=0}^{\infty} \mathbb{E}\left[\sum_{x \in \tilde{\mathcal{G}}_{n}} e^{-\zeta S_{x}} \sum_{y \in \tilde{\mathcal{G}}_{1}^{x}}\left(V_{\theta}^{x y}+1\right)\right]
$$

and condition first on $\mathcal{F}_{n+1}$ to obtain

$$
\mathbb{E}\left[\sum_{x \in \tilde{\mathcal{P}}} e^{-\zeta S_{x}} \sum_{y \in \tilde{\mathcal{G}}_{1}^{x}}\left(V_{\theta}^{x y}+1\right)\right]=\mathbb{E}\left[V_{\theta}+1\right] \sum_{n=0}^{\infty} \mathbb{E}\left[\sum_{x \in \tilde{\mathcal{G}}_{n}} e^{-\zeta S_{x}} \tilde{\nu}_{x}\right],
$$

since the $V_{\theta}^{x y}$ are independent of $\mathcal{F}_{n+1}$ and all have the distribution of $V_{\theta}$. Now condition on $\mathcal{F}_{n}$ to obtain

$$
\begin{aligned}
\mathbb{E}\left[\sum_{x \in \tilde{\mathcal{P}}} e^{-\zeta S_{x}} \sum_{y \in \tilde{\mathcal{G}}_{1}^{x}}\left(V_{\theta}^{x y}+1\right)\right] & =\left(\mathbb{E}\left[V_{\theta}\right]+1\right) \mathbb{E}[\tilde{\nu}] \sum_{n=0}^{\infty} \mathbb{E}\left[\sum_{x \in \tilde{\mathcal{G}}_{n}} e^{-\zeta S_{x}}\right] \\
& =\frac{\left(\mathbb{E}\left[V_{\theta}\right]+1\right) \mathbb{E}[\tilde{\nu}]}{[1-\tilde{m}(\zeta)]_{+}}
\end{aligned}
$$

Similarly, $\mathbb{E}\left[V_{\theta}\right]=\mathbb{E}[\nu][1-m(\theta)]_{+}^{-1}$. Since $\tilde{m}(\tilde{D}+\delta)<1$ and $m(D+\delta)<1$,

$$
\mathbb{E}\left[\sum_{x \in \tilde{\mathcal{P}}} e^{-\zeta S_{x}} \sum_{y \in \tilde{\mathcal{G}}_{1}^{x}}\left(V_{\theta}^{x y}+1\right)\right]<\infty
$$

for $\zeta=\tilde{D}+\delta$ and $\theta=D+\delta$. This, in conjunction with (3.23) and Claim 2, completes the proof in the case $p=1$. 
If $p>1$, then $\left\{\tilde{\mathcal{G}}_{n}: n \geq 0\right\}$ is not a Galton-Watson process anymore. However, $\left\{\mathcal{G}_{n}^{(p)}: n \geq 0\right\}$ is, where $\mathcal{G}_{0}^{(p)}=\{o\}$ and, for $n \geq 1, \mathcal{G}_{n}^{(p)}$ consists of those sequences in $\mathcal{G}_{n p}$ for which, for all $1 \leq k \leq n$, there exists a $v \in \phi_{x_{1} \ldots x_{(k-1) p+1}} \circ \ldots \circ \phi_{x_{1} \ldots x_{k p}}(J)$, with $\operatorname{dist}\left(v, G^{c}\right) \leq \alpha$. Furthermore, $\tilde{\mathcal{G}}_{n p} \subseteq \mathcal{G}_{n}^{(p)}$, for all $n$.

Inequality (3.23) is still valid and it only remains to show that the expectation of the right-hand side of (3.23) is finite. Conditioning again first on $\mathcal{F}_{n+1}$ and then on $\mathcal{F}_{n}$, we have successively

$$
\begin{aligned}
\mathbb{E}\left[\sum_{x \in \tilde{\mathcal{P}}} e^{-\zeta S_{x}} \sum_{y \in \tilde{\mathcal{G}}_{1}^{x}}\left(V_{\theta}^{x y}+1\right)\right] & =\left(\mathbb{E}\left[V_{\theta}\right]+1\right) \sum_{n=0}^{\infty} \mathbb{E}\left[\sum_{x \in \tilde{\mathcal{G}}_{n}} e^{-\zeta S_{x}} \sum_{y \in \tilde{\mathcal{G}}_{1}^{x}} 1\right] \\
& \leq\left(\mathbb{E}\left[V_{\theta}\right]+1\right) \mathbb{E}[\nu] \sum_{n=0}^{\infty} \mathbb{E}\left[\sum_{x \in \tilde{\mathcal{G}}_{n}} e^{-\zeta S_{x}}\right] .
\end{aligned}
$$

Next observe that

$$
\begin{aligned}
\sum_{n=0}^{\infty} \mathbb{E}\left[\sum_{x \in \tilde{\mathcal{G}}_{n}} e^{-\zeta S_{x}}\right] & =\sum_{n=0}^{\infty} \mathbb{E}\left[\sum_{x \in \tilde{\mathcal{G}}_{n p}} \sum_{k=0}^{p-1} \sum_{y \in \tilde{\mathcal{G}}_{k}^{x}} e^{-\zeta S_{x}} e^{-\zeta\left(S_{x y}-S_{x}\right)}\right] \\
& \leq \sum_{n=0}^{\infty} \sum_{k=0}^{p-1}[m(\zeta)]^{k} \mathbb{E}\left[\sum_{x \in \mathcal{G}_{n}^{(p)}} e^{-\zeta S_{x}}\right] \\
& =\frac{[m(\zeta)]^{p}-1}{m(\zeta)-1} \sum_{n=0}^{\infty}\left(\mathbb{E}\left[\sum_{x \in \mathcal{G}_{1}^{(p)}} e^{-\zeta S_{x}}\right]\right)^{n} .
\end{aligned}
$$

Now take $\zeta=\tilde{D}+\delta$ and $\theta=D+\delta$ again, observe that

$$
\mathbb{E}\left[\sum_{x \in \mathcal{G}_{1}^{(p)}} e^{-\zeta S_{x}}\right]=\mathbb{E}\left[\sum_{x \in \tilde{\mathcal{G}}_{p}} e^{-\zeta S_{x}}\right]<1
$$

for $\zeta=\tilde{D}+\delta$, by (3.18), and conclude the proof as in the case $p=1$.

We now conclude the proof of Lemma 3.6. Recall 3.7).

$$
R_{t}=\sum_{x \in \mathcal{G}_{1}}\left|K_{x}\left(e^{-t}\right)\right|-\left|K\left(e^{-t}\right)\right| \leq \sum_{x \in \mathcal{G}_{1}} \sum_{y \in \mathcal{G}_{1} \backslash\{x\}}\left|K_{x}\left(e^{-t}\right) \cap K_{y}\left(e^{-t}\right)\right|,
$$

where recall $K_{x}=K \cap \phi_{x}(J)$. Let $Q_{x}(2 \epsilon)$ denote the maximum cardinality of a $2 \epsilon$-separated subset $F_{x}$ of $K_{x}$, such that dist $\left(v, \phi_{x}\left(G^{c}\right)\right) \leq 2 \epsilon$ for all $v \in F_{x}$. Then,

$$
\bigcup_{y \in \mathcal{G}_{1} \backslash\{x\}} K_{x}\left(e^{-t}\right) \cap K_{y}\left(e^{-t}\right) \subseteq \bigcup_{v \in F_{x}} B_{3 \epsilon^{-t}}(v),
$$

so that

$$
R_{t} \leq \sum_{x \in \mathcal{G}_{1}} Q_{x}\left(2 e^{-t}\right)\left(3 e^{-t}\right)^{d} c_{d}
$$

Now, given that $x \in \mathcal{G}_{1}, Q_{x}(2 \epsilon)=Q^{x}\left(2 \epsilon e^{S_{x}}\right)$, where for $\beta>0, Q^{x}(\beta)$ is the maximum cardinality of a $\beta$-separated subset $F^{x}$ of $K^{x}=\phi_{x}^{-1}\left(K_{x}\right)$, such that 
$\operatorname{dist}\left(v, G^{c}\right) \leq \beta$ for all $v \in F^{x}$. Furthermore, $Q^{x}(\beta)$ has the same distribution as $Q(\beta)$, for each $\beta>0$. Hence

$$
R_{t} \leq c_{d} 3^{d} e^{-d t} \sum_{x \in \mathcal{G}_{1}} Q^{x}\left(2 e^{-t+S_{x}}\right)
$$

and therefore, for any $0<\delta \leq(D-\tilde{D}) / 2$,

$$
\sup _{t \geq 0} e^{(d-D+\delta) t} R_{t} \leq c_{d} 3^{d} 2^{-(\tilde{D}+\delta)} \sum_{x \in \mathcal{G}_{1}} e^{-(\tilde{D}+\delta) S_{x}} \sup _{0<\epsilon \leq 2 e^{S_{x}}} \epsilon^{\tilde{D}+\delta} Q^{x}(\epsilon) .
$$

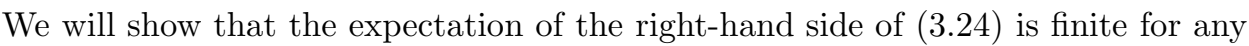
such $\delta$ and this will prove Lemma 3.6 with $h(t)=e^{-\delta t} \mathbf{1}_{[0, \infty)}(t)$.

Since $J$ is compact and $K^{x} \subseteq J$, for all $x \in \mathcal{G}_{1}$, there exists a finite deterministic constant $C$, such that $Q^{x}(\epsilon) \leq C$ for all $\epsilon>\alpha$ and all $x \in \mathcal{G}_{1}$. Thus

$$
\sum_{x \in \mathcal{G}_{1}} e^{-(\tilde{D}+\delta) S_{x}} \sup _{0<\epsilon \leq 2 e^{S_{x}}} \epsilon^{\tilde{D}+\delta} Q^{x}(\epsilon) \leq \sum_{x \in \mathcal{G}_{1}} e^{-(\tilde{D}+\delta) S_{x}} \sup _{0<\epsilon \leq \alpha} \epsilon^{\tilde{D}+\delta} Q^{x}(\epsilon)+\nu 2^{\tilde{D}+\delta} C,
$$

and since $\mathbb{E}[\nu]$ is finite, it suffices to show that

$$
\mathbb{E}\left[\sum_{x \in \mathcal{G}_{1}} e^{-(\tilde{D}+\delta) S_{x}} \sup _{0<\epsilon \leq \alpha} \epsilon^{\tilde{D}+\delta} Q^{x}(\epsilon)\right]<\infty .
$$

But, by conditioning on $\mathcal{F}_{1}$,

$$
\begin{aligned}
\mathbb{E}\left[\sum_{x \in \mathcal{G}_{1}} e^{-(\tilde{D}+\delta) S_{x}} \sup _{0<\epsilon \leq \alpha} \epsilon^{\tilde{D}+\delta} Q^{x}(\epsilon)\right] & =\mathbb{E}\left[\sup _{0<\epsilon \leq \alpha} \epsilon^{\tilde{D}+\delta} Q(\epsilon)\right] \mathbb{E}\left[\sum_{x \in \mathcal{G}_{1}} e^{-(\tilde{D}+\delta) S_{x}}\right] \\
& =\mathbb{E}\left[\sup _{0<\epsilon \leq \alpha} \epsilon^{\tilde{D}+\delta} Q(\epsilon)\right] m(\tilde{D}+\delta)
\end{aligned}
$$

and this is finite by Claim 3 .

3.4. Convergence in mean. Theorem 3.1 deals with the almost sure limiting behavior of the quantities $\epsilon^{-(d-D)}|K(\epsilon)|$ and $T^{-1} \int_{0}^{T} e^{(d-D) t}\left|K\left(e^{-t}\right)\right| d t$. One can obtain results concerning the limiting behavior of the means of these quantities via the ordinary renewal theorem, as in the deterministic case.

Recall (3.7), (3.8) and (3.9). The function $w(t)=\mathbb{E}\left[W_{t}\right]$ satisfies the ordinary renewal equation

$$
w=f+w * F,
$$

where $*$ denotes convolution, $F$ is the probability distribution function

$$
F(t)=\mathbb{E}\left[\sum_{x \in \mathcal{G}_{1}} e^{-D S_{x}} \mathbf{1}_{(-\infty, t]}\left(S_{x}\right)\right]
$$

and $f(t)=e^{-D t} \mathbb{E}\left[Z_{t}\right]$.

Now assume the hypotheses of Theorem 3.1 hold. By (3.11) we have that

$$
\sum_{k=1}^{\infty} \sup _{s \in[k-1, k]}|f(s)|<\infty .
$$

Furthermore, $f$ is continuous except at a countable number of points. This is because the sample paths of $Z$ have left- and right-hand limits and hence, by the 
dominated convergence theorem (use (3.11) to dominate), $f$ has left- and righthand limits. It now follows that $f$ is directly Riemann integrable over $[0, \infty)$. In particular $f$ is also bounded.

By (3.16), $w$ is bounded on finite intervals and therefore $w=f * U$, where $U=\sum_{n=0}^{\infty} F^{* n}$. By the ordinary renewal theorem then

$$
\mathbb{E}\left[W_{t}\right] \longrightarrow \frac{1}{-m^{\prime}(D)} \int_{0}^{\infty} e^{-D s} \mathbb{E}\left[Z_{s}\right] d s,
$$

as $t \longrightarrow \infty$, if $A \mapsto \mathbb{E}[\xi(A)]$ is non-lattice, while in the lattice case

$$
\mathbb{E}\left[W_{s+n \lambda}\right] \longrightarrow \frac{\lambda e^{-D s}}{-m^{\prime}(D)} \sum_{k=0}^{\infty} e^{-D k \lambda} \mathbb{E}\left[Z_{s+k \lambda}\right]
$$

as $n \longrightarrow \infty$, for each $s \in[0, \lambda)$. As in the proof of Theorem 2.3 then, one always has that, as $T \longrightarrow \infty$,

$$
\frac{1}{T} \int_{0}^{T} \mathbb{E}\left[W_{t}\right] d t \longrightarrow \frac{1}{-m^{\prime}(D)} \int_{0}^{\infty} e^{-D s} \mathbb{E}\left[Z_{s}\right] d s .
$$

We summarize the above in the following theorem. Recall that $M_{1}=\sum_{x \in \mathcal{G}_{1}} r_{x}^{D}$.

Theorem 3.7. (i) Under the hypotheses of Theorem [3.1]

$$
\frac{1}{T} \int_{0}^{T} e^{(d-D) t} \mathbb{E}\left[\left|K\left(e^{-t}\right)\right|\right] d t \longrightarrow \frac{1}{-m^{\prime}(D)} \int_{0}^{\infty} e^{-D s} \mathbb{E}\left[Z_{s}\right] d s,
$$

as $T \longrightarrow \infty$; if in addition $A \mapsto \mathbb{E}[\xi(A)]$ is non-lattice, then also, as $\epsilon \rightarrow 0$,

$$
\epsilon^{-(d-D)} \mathbb{E}[|K(\epsilon)|] \longrightarrow \frac{1}{-m^{\prime}(D)} \int_{0}^{\infty} e^{-D s} \mathbb{E}\left[Z_{s}\right] d s .
$$

(ii) If in addition $\mathbb{E}\left[M_{1} \log ^{+} M_{1}\right]<\infty$, then both, the convergence in Theorem 3.1 $\mathrm{i}$ ) (in the non-lattice case) and the convergence in Theorem 3.1(ii), hold also in $L^{1}$.

Proof. Assertion (i) has already been established above. Under the additional assumption $\mathbb{E}\left[M_{1} \log ^{+} M_{1}\right]<\infty$, we also have, by Theorem 3.3 , that $\mathbb{E}\left[M_{\infty}\right]=1$ and so $\mathbb{E}\left[W_{t}\right] \longrightarrow \mathbb{E}\left[\lim W_{t}\right]$, in the non-lattice case, where $\lim W_{t}$ denotes the almost sure limit in (i) of Theorem 3.1 This, in the presence of the almost sure convergence of Theorem 3.1 (i) and non-negativity of $W_{t}$, implies the $L^{1}$-convergence in the non-lattice case (see Billingsley [4, Corollary following Theorem 16.14).

Similarly, to establish the $L^{1}$-convergence of $T^{-1} \int_{0}^{T} W_{t} d t$ to its a.s.-limit, observe that by (i) of the present theorem, Theorem 3.1(ii) and the fact that $\mathbb{E}\left[M_{\infty}\right]=$ 1 , we have

$$
\mathbb{E}\left[\frac{1}{T} \int_{0}^{T} W_{t} d t\right]=\frac{1}{T} \int_{0}^{T} \mathbb{E}\left[W_{t}\right] d t \longrightarrow \mathbb{E}\left[\lim _{T \longrightarrow \infty} \frac{1}{T} \int_{0}^{T} W_{t} d t\right] .
$$

Again this, in the presence of the corresponding a.s.-convergence and non-negativity, implies convergence in $L^{1}$. 
3.5. Proof of Theorem 3.2. (i) When $\mathbb{E}\left[M_{1} \log ^{+} M_{1}\right]=\infty$, we have that $M_{\infty}=0$ with probability equal to 1 , by Theorem 3.3 so $L=0$ with probability equal to 1 , by the representation $L=M_{\infty} \times$ constant (see (3.12) and (3.15)).

(ii) Assume now $\mathbb{E}\left[M_{1} \log ^{+} M_{1}\right]<\infty$. By our assumption (3.2), namely that $\mathbb{P}(K \cap G \neq \emptyset)>0$, there exist a $\delta>0$, and an event (i.e., a measurable set) $A_{\delta}$, such that $\mathbb{P}\left(A_{\delta}\right)>0$ and

$$
\text { for } \omega \in A_{\delta} \exists v(\omega) \in K(\omega) \text { s.t. } B_{\delta}(v(\omega)) \subset G \text {, }
$$

where, recall, $B_{\delta}(v)$ denotes the ball with center $v$ and radius $\delta$. Fix this $\delta$ for the rest of the proof.

Next fix $a>0$, to be specified later in the proof. We claim that, on $A_{\delta}$,

$$
\left|K\left(\delta e^{-t-a}\right)\right| \geq c_{d}\left(\delta e^{-t-a}\right)^{d} \sum_{x \in \mathcal{P}_{t}} \mathbf{1}_{(t, t+a]}\left(S_{x}\right),
$$

where, recall,

$$
\mathcal{P}_{t}=\left\{x=x_{1} \ldots x_{n} \in \mathcal{P}: S_{x_{1} \ldots x_{n-1}} \leq t<S_{x_{1} \ldots x_{n}}\right\} .
$$

This is because, for distinct $x=x_{1} \ldots x_{n}$ and $y=y_{1} \ldots y_{m}$ in $\mathcal{P}_{t}$, we must have $x_{p} \neq y_{p}$ for some $p \leq \min \{n, m\}$; then however,

$$
\phi_{x_{1}} \circ \ldots \circ \phi_{x_{1} \ldots x_{n}}(G) \cap \phi_{y_{1}} \circ \ldots \circ \phi_{y_{1} \ldots y_{m}}(G)=\emptyset .
$$

On $A_{\delta}$, we may choose a point $v \in K$ such that $B_{\delta}(v) \subset G$, and then

$$
B_{\delta e^{-S_{x}}}\left(v_{x}\right) \cap B_{\delta e^{-S_{y}}}\left(v_{y}\right)=\emptyset,
$$

where $v_{x}=\phi_{x_{1}} \circ \ldots \circ \phi_{x_{1} \ldots x_{n}}(v)$ and $v_{y}=\phi_{y_{1}} \circ \ldots \circ \phi_{y_{1} \ldots y_{m}}(v)$. But if $x \in \mathcal{P}_{t}$, with $S_{x} \leq t+a$, then $\delta e^{-t-a} \leq \delta e^{-S_{x}}$, and so the balls $B_{\delta e^{-t-a}}\left(v_{x}\right), x \in \mathcal{P}_{t}$ with $t<S_{x} \leq t+a$, are disjoint. This proves (3.26).

Now write

$$
\sum_{x \in \mathcal{P}_{t}} \mathbf{1}_{(t, t+a]}\left(S_{x}\right)=\sum_{x \in \mathcal{P}} Z_{t-S_{x}}^{x}
$$

with $Z_{t}=\xi(t, t+a]$, if $t \geq 0$, and $Z_{t}=0$ otherwise, where recall $\xi(A)=$ $\sum_{x \in \mathcal{G}_{1}} \mathbf{1}_{A}\left(S_{x}\right)$. We may apply Theorem 3.4 since obviously $\sup _{t \geq 0} Z_{t} \leq \nu$, and condition (3.4) holds with $h(t)=e^{-D t} \mathbf{1}_{[0, \infty)}(t)$. We conclude that, in the nonlattice case, with probability equal to 1 ,

$$
e^{-D t} \sum_{x \in \mathcal{P}_{t}} \mathbf{1}_{(t, t+a]}\left(S_{x}\right) \longrightarrow \frac{M_{\infty}}{-m^{\prime}(D)} \int_{0}^{\infty} e^{-D u} \mathbb{E}[\xi(u, u+a]] d u .
$$

Write the limit above as $M_{\infty} C$, and notice that

$$
\begin{gathered}
C=\frac{1}{-m^{\prime}(D)} \mathbb{E}\left[\sum_{x \in \mathcal{G}_{1}} \int_{\max \left\{0, S_{x}-a\right\}}^{S_{x}} e^{-D u} d u\right] \\
=\frac{1}{-D m^{\prime}(D)} \mathbb{E}\left[\sum_{x \in \mathcal{G}_{1}}\left\{\left(e^{D a}-1\right) e^{-D S_{x}} \mathbf{1}_{(a, \infty)}\left(S_{x}\right)+\left(1-e^{-D S_{x}}\right) \mathbf{1}_{(0, a]}\left(S_{x}\right)\right\}\right],
\end{gathered}
$$

So

$$
C>0
$$

for whatever choice of $a>0$ we make; so fix some $a>0$ for definiteness. 
Now note that the convergence in (3.27) also holds in $L^{1}$. To see this let $x(t)$ denote the expected value of $e^{-D t} \sum_{x \in \mathcal{P}_{t}} \mathbf{1}_{(t, t+a]}\left(S_{x}\right)$, for $t \geq 0$, and note that it satisfies the ordinary renewal equation $x=f+x * F$, where $F$ is the distribution function defined in (3.25), and $f(t)=\mathbb{E}[\xi(t, t+a]]$, for $t \geq 0$. It follows, by the (ordinary) renewal theorem that

$$
e^{-D t} \mathbb{E}\left[\sum_{x \in \mathcal{P}_{t}} \mathbf{1}_{(t, t+a]}\left(S_{x}\right)\right] \longrightarrow C
$$

It now follows by (3.27), 3.29) and non-negativity, that the convergence in (3.27) also holds in $L^{1}$ (see Billingsley 4, Corollary following Theorem 16.14) and therefore we also have

$$
\epsilon^{-D t} \mathbb{E}\left[\mathbf{1}_{A_{\delta}} \sum_{x \in \mathcal{P}_{t}} \mathbf{1}_{(t, t+a]}\left(S_{x}\right)\right] \longrightarrow C \mathbb{E}\left[M_{\infty} \mathbf{1}_{A_{\delta}}\right]
$$

Note that, since $A_{\delta} \subseteq\{K \neq \emptyset\}$ and $\mathbb{P}\left(M_{\infty}>0 \mid K \neq \emptyset\right)=1$, we have that $M_{\infty}>0$ almost surely on $A_{\delta}$. Thus

$$
\mathbb{E}\left[M_{\infty} \mathbf{1}_{A_{\delta}}\right]>0,
$$

since we chose $\delta$ so that $\mathbb{P}\left(A_{\delta}\right)>0$. Combining this with (3.26), 3.28) and (3.30) we then have that

$$
\begin{aligned}
\lim _{\epsilon \rightarrow 0} \epsilon^{-(d-D)} \mathbb{E}[|K(\epsilon)|] & \geq \liminf _{t \rightarrow \infty} e^{(d-D)(t+a)} \delta^{-(d-D)} \mathbb{E}\left[\mathbf{1}_{A_{\delta}}\left|K\left(\delta e^{-t-a}\right)\right|\right] \\
& \geq c_{d} \delta^{D} e^{-D a} \lim _{t \rightarrow \infty} e^{-t D} \mathbb{E}\left[\mathbf{1}_{A_{\delta}} \sum_{x \in \mathcal{P}_{t}} \mathbf{1}_{(t, t+a]}\left(S_{x}\right)\right] \\
& =c_{d} \delta^{D} e^{-D a} C \mathbb{E}\left[M_{\infty} \mathbf{1}_{A_{\delta}}\right]>0 .
\end{aligned}
$$

However, by Theorem 3.7 and (3.12), and since we are assuming $\mathbb{E}\left[M_{1} \log ^{+} M_{1}\right]<$ $\infty$, we have that

$$
L=\lim _{\epsilon \rightarrow 0} \epsilon^{-(d-D)}|K(\epsilon)|=M_{\infty} \lim _{\epsilon \rightarrow 0} \epsilon^{-(d-D)} \mathbb{E}[|K(\epsilon)|]
$$

and we conclude that $L>0$ iff $M_{\infty}>0$ in the non-lattice case. The result follows from Theorem 3.3 .

For the lattice case choose $a=k \lambda$, where $\lambda>0$ is the span and $k$ is such that $\mathbb{P}\left(S_{x}=k \lambda\right.$ some $\left.x \in \mathcal{G}_{1}\right)>0$, but $\mathbb{P}\left(S_{x}=j \lambda\right.$ some $\left.x \in \mathcal{G}_{1}\right)=0$ for all $0 \leq j<k$. Theorem 3.4 implies that, for any fixed $s \in[0, \lambda)$, as $n \longrightarrow \infty$,

$$
e^{-D n \lambda} \sum_{x \in \mathcal{P}_{s+n \lambda}} \mathbf{1}_{(s+n \lambda, s+n \lambda+a]}\left(S_{x}\right) \longrightarrow \frac{\lambda M_{\infty}}{-m^{\prime}(D)} \sum_{i=0}^{\infty} e^{-D \lambda i} \mathbb{E}[\xi(s+i \lambda, s+i \lambda+a]],
$$


with probability equal to 1 . Write again the limit as $M_{\infty} C(s)$; by our choice of $a$

$$
\begin{aligned}
C(s) & =\frac{\lambda}{-m^{\prime}(D)} \mathbb{E}\left[\sum_{x \in \mathcal{G}_{1}} \sum_{\max \left\{0, S_{x}-a-s\right\} \leq n \lambda<S_{x}-s} e^{-D n \lambda}\right] \\
& \geq \frac{\lambda}{-m^{\prime}(D)} \mathbb{E}\left[\sum_{x \in \mathcal{G}_{1}} \mathbf{1}_{\{k \lambda\}}\left(S_{x}\right) \sum_{\max \left\{0, S_{x}-a-s\right\} \leq n \lambda<S_{x}-s} e^{-D n \lambda}\right] \\
& =\frac{\lambda}{-m^{\prime}(D)} \frac{1-e^{-k D \lambda}}{1-e^{-D \lambda}} \mathbb{E}[\xi(\{k \lambda\})],
\end{aligned}
$$

for $s \in[0, \lambda)$. Let $C$ denote the last expression above; then

$$
C(s) \geq C>0,
$$

for all $s \in[0, \lambda)$. As in the non-lattice case, the convergence in (3.32) also holds in $L^{1}$ and therefore, as $n \longrightarrow \infty$,

$$
e^{-D n \lambda} \mathbb{E}\left[\mathbf{1}_{A_{\delta}} \sum_{x \in \mathcal{P}_{s+n \lambda}} \mathbf{1}_{(s+n \lambda, s+n \lambda+a]}\left(S_{x}\right)\right] \longrightarrow C(s) \mathbb{E}\left[M_{\infty} \mathbf{1}_{A_{\delta}}\right],
$$

for each $s \in[0, \lambda)$. Now

$$
\begin{aligned}
& \inf _{s \in[0, \lambda)} \lim _{n \rightarrow \infty} e^{(s+n \lambda)(d-D)} \mathbb{E}\left[\left|K\left(e^{-s-n \lambda}\right)\right|\right] \\
= & \inf _{s \in[0, \lambda)} \lim _{n \rightarrow \infty} \delta^{-(d-D)} e^{(s+n \lambda+a)(d-D)} \mathbb{E}\left[\left|K\left(\delta e^{-s-n \lambda-a}\right)\right|\right] \\
\geq & \inf _{s \in[0, \lambda)} \lim _{n \rightarrow \infty} \delta^{-(d-D)} e^{(s+n \lambda+a)(d-D)} \mathbb{E}\left[\mathbf{1}_{A_{\delta}}\left|K\left(\delta e^{-s-n \lambda-a}\right)\right|\right]
\end{aligned}
$$

and we then have successively by (3.26), (3.34), (3.33) and (3.31)

$$
\begin{aligned}
& \inf _{s \in[0, \lambda)} \lim _{n \rightarrow \infty} e^{(s+n \lambda)(d-D)} \mathbb{E}\left[\left|K\left(e^{-s-n \lambda}\right)\right|\right] \\
\geq & c_{d} \delta^{D} e^{-a D} \inf _{s \in[0, \lambda)} \lim _{n \rightarrow \infty} e^{-(s+n \lambda) D} \mathbb{E}\left[\mathbf{1}_{A_{\delta}} \sum_{x \in \mathcal{P}_{s+n \lambda}} \mathbf{1}_{(s+n \lambda, s+n \lambda+a]}\left(S_{x}\right)\right] \\
= & c_{d} \delta^{D} e^{-a D} \mathbb{E}\left[M_{\infty} \mathbf{1}_{A_{\delta}}\right] \inf _{s \in[0, \lambda)} e^{-D s} C(s) \\
\geq & c_{d} \delta^{D} e^{-(a+\lambda) D} \mathbb{E}\left[M_{\infty} \mathbf{1}_{A_{\delta}}\right] C>0 .
\end{aligned}
$$

But by Theorem 3.7 again, and (3.15),

$$
\begin{aligned}
L & =\lim _{T \rightarrow \infty} \frac{1}{T} \int_{0}^{T} e^{(d-D) t}\left|K\left(e^{-t}\right)\right| d t \\
& =M_{\infty} \lim _{T \rightarrow \infty} \frac{1}{T} \int_{0}^{T} e^{(d-D) t} \mathbb{E}\left[\left|K\left(e^{-t}\right)\right|\right] d t \\
& =M_{\infty} \frac{1}{\lambda} \int_{0}^{\lambda} \lim _{n \rightarrow \infty} e^{(s+n \lambda)(d-D)} \mathbb{E}\left[\left|K\left(e^{-s-n \lambda}\right)\right|\right] d s \\
& \geq M_{\infty} \inf _{s \in[0, \lambda)} \lim _{n \rightarrow \infty} e^{(s+n \lambda)(d-D)} \mathbb{E}\left[\left|K\left(e^{-s-n \lambda}\right)\right|\right]
\end{aligned}
$$

and we conclude that $L>0$ iff $M_{\infty}>0$ for the lattice case as well. By Theorem 3.3 this concludes the proof of the theorem. 


\section{EXAMPLES}

4.1. Cantor set. As already mentioned after Theorem 2.3 one cannot apply part (i) of this theorem to the usual ternary Cantor set. For this set $r_{1}=r_{2}=1 / 3$ and so $\left\{-\log r_{1},-\log r_{2}\right\} \subset \lambda \mathbb{Z}$, with $\lambda=\log 3$; here $D=\log _{3} 2$. Using (2.9), one can actually compute the limits

$$
L(s)=\lim _{n \rightarrow \infty} e^{(1-D)(s+n \lambda)}\left|K\left(e^{-s-n \lambda}\right)\right|,
$$

along sequences $s+n \lambda, s \in[0, \log 3)$; one finds

$$
L(s)= \begin{cases}e^{(1-D) s}\left(\frac{3}{2}+e^{-s}\right) & \text { for } 0 \leq s<\log 2 \\ e^{(1-D) s}\left(1+2 e^{-s}\right) & \text { for } \log 2 \leq s<\log 3 .\end{cases}
$$

Thus $\epsilon^{-(d-D)}|K(\epsilon)|$ does not have a limit in this case.

(For the usual ternary Cantor set this fact is well known and can be obtained by direct computation, without the aid of Theorem 2.3. see for example [15]; see also the Acknowledgments at the end of section 1.)

4.2. Fractal percolation (Mandelbrot's canonical curdling). Let $d \geq 1$ and fix an integer $N \geq 2$ and $p \in(0,1)$, with $N^{d} p>1$. We consider the random set $K$ obtained as follows. Divide the unit (hyper)cube $[0,1]^{d}$ into the $N^{d}$ congruent subcubes $\prod_{k=1}^{d}\left[i_{k} N^{-1},\left(i_{k}+1\right) N^{-1}\right], 0 \leq i_{k}<N, 1 \leq k \leq d$, and keep each of them with probability $p$ (discard it with probability $1-p$ ), independently of all other subcubes. Next divide each of the subcubes kept in the first step into the $N^{d}$ congruent subcubes of side-length $N^{-2}$ it contains and keep each of these with probability $p$, independently of all else. The set $K$ is then obtained by continuing this process ad-infinitum.

The set $K$ is a stochastically self-similar set as described in subsection 3.1, with $J=[0,1]^{d}, G=(0,1)^{d}$ and $\Phi=\left\{\phi_{1}, \ldots, \phi_{\nu}\right\}$ such that each $\phi_{i}$ is one of the similarities

$$
\psi_{i_{1} \ldots i_{d}}(v)=\frac{1}{N}\left[v+\left(i_{1}, \ldots, i_{d}\right)^{T}\right], \quad v \in \mathbb{R}^{d}, \quad 0 \leq i_{k}<N, \quad 1 \leq k \leq d,
$$

where $T$ denotes transpose. In this case $\mathbb{E}[\nu]=N^{d} p$ and this explains our assumption $N^{d} p>1$. The dimension of $K$, almost surely on the event $\{K \neq \emptyset\}$, is the solution in $D$ of the equation

$$
1=\mathbb{E}\left[\sum_{x \in \mathcal{G}_{1}} e^{-D S_{x}}\right]=N^{-D} \mathbb{E}[\nu] ;
$$

so $D=d+\log _{N} p$. Furthermore, the measure $A \mapsto \mathbb{E}[\xi(A)]$ is lattice, with span $\lambda=\log N$, and Theorem 3.1(ii) applies, as clearly

$$
\mathbb{P}(K \cap G \neq \emptyset)>0 .
$$

Note that the assumption that we keep subcubes independently of one-another is irrelevant to this discussion and we could have any other distribution for $\Phi$, provided (4.1) is satisfied (independence between successive steps is still required however); the value of $\mathbb{E}[\nu]$, and hence also of $D$, would then be different of course.

Note also that, because $\nu$ is bounded here, Theorem 3.7](ii) also applies. 
4.3. Level-sets of stable processes. Let $\left\{X_{t}: t \geq 0\right\}$ be a real-valued stable process of index $\alpha \in(0,2]$, started at 0 . This is a process with stationary and independent increments (Levy process), which has the following scaling property: for every $s>0$, the process $\left\{s^{-1 / \alpha} X_{s t}: t \geq 0\right\}$ has the same law (distribution) as $X$ itself. For $\alpha=2$, the process $X$ is a constant multiple of standard Brownian motion.

Let $K=\left\{s \geq 0: X_{s}=0\right\}$, the zero-set of $X$, and $K_{t}=K \cap[0, t], t \geq 0$, the zero-set of $X$ up to time $t$. It is well known that, when $1<\alpha \leq 2, K$ and each $K_{t}$ are nonempty closed perfect sets (because 0 is regular for itself, by the scaling property), of Hausdorff dimension $D=1-1 / \alpha$ ([5], 24]). When $0<\alpha \leq 1$ on the other hand, $K=\{0\}$, as singletons are polar in this case (see [2]). We therefore restrict attention to the case $1<\alpha \leq 2$ henceforth. We will explain how to apply Theorems 3.1 and 3.7 to obtain the following statement: for each fixed $t \geq 0$, the limit

$$
L_{t}=\lim _{\epsilon \rightarrow 0} \epsilon^{-1 / \alpha}\left|K_{t}(\epsilon)\right|
$$

exists a.s. and this convergence also holds in $L^{1}$.

Fix $t>0$ and let $\left\{Y_{s}: 0 \leq s \leq t\right\}$ be the corresponding stable bridge; this, intuitively, is the process $\left.X\right|_{[0, t]}$ conditioned to be 0 at time $t$. Denote by $K_{Y}$ the zero set of $Y: K_{Y}=\left\{s \in[0, t]: Y_{s}=0\right\}$. The set $K_{Y}$ is then a stochastically self-similar set in the sense of subsection 3.1, with $J=[0, t]$ and $\Phi=\left\{\phi_{1}, \phi_{2}\right\}$, where $\phi_{1}$ and $\phi_{2}$ are the similarities of $\mathbb{R}$ given by

$$
\phi_{1}(v)=\frac{\tau_{1}}{t} v, \quad \phi_{2}(v)=\frac{\tau_{2}}{t} v+t-\tau_{2},
$$

and $\tau_{1}=\sup \left\{s \in[0, t / 2]: Y_{s}=0\right\}$ and $t-\tau_{2}=\inf \left\{s \in[t / 2, t]: Y_{s}=0\right\}$. Clearly, condition (3.2) is satisfied, as, for example, $\tau_{1} \in K \cap G$. Also, the measure $A \mapsto$ $\mathbb{E}[\xi(A)]$ is non-lattice, as $\tau_{1}$ and $\tau_{2}$ have (actually identical) absolutely continuous distributions. Finally, $K_{Y}$ also has dimension $1-1 / \alpha$. One way to see this is to observe that

$$
K_{Y} \stackrel{\mathcal{L}}{=} \frac{t}{\tau} K_{t},
$$

where $\stackrel{\mathcal{L}}{=}$ means 'equal in law' and $\tau=\sup \left\{s \in[0, t]: X_{s}=0\right\}$. This is because the processes $Y$ and $\left\{(\tau / t)^{-1 / \alpha} X_{s \tau / t}: 0 \leq s \leq t\right\}$ have the same laws (i.e., the latter is a bridge), by the scaling property (see [2], Theorem VIII.12). It now follows from Theorem 3.1 that, with probability equal to one, the limit

$$
L_{Y}=\lim _{\epsilon \rightarrow 0} \epsilon^{-1 / \alpha}\left|K_{Y}(\epsilon)\right|
$$

exists and this convergence also holds in $L^{1}$, by Theorem 3.7. Statement (4.2) then follows from this convergence, using the representation (4.3) and observing that

$$
\begin{aligned}
\lim _{\epsilon \rightarrow 0} \epsilon^{-1 / \alpha}\left|K_{t}(\epsilon)\right| & =\lim _{\epsilon \rightarrow 0} \epsilon^{-1 / \alpha} \frac{\tau}{t}\left|\left(\frac{t}{\tau} K_{t}\right)\left(\frac{\epsilon t}{\tau}\right)\right| \\
& =\left(\frac{\tau}{t}\right)^{1-1 / \alpha} \lim _{\epsilon \rightarrow 0}\left(\frac{\epsilon t}{\tau}\right)^{-1 / \alpha}\left|\left(\frac{t}{\tau} K_{t}\right)\left(\frac{\epsilon t}{\tau}\right)\right| .
\end{aligned}
$$

As a matter of fact, taking $Y_{s}=(\tau / t)^{-1 / \alpha} X_{s \tau / t}, 0 \leq s \leq t$, as we may, we obtain that $L_{t}=(\tau / t)^{1-1 / \alpha} L_{Y}$; furthermore $\tau$ is independent of $L_{Y}$ ([2], Theorem VIII.12). 
Clearly, one can then deal similarly with the case where the starting point $X_{0}$ is an arbitrary point $x \in \mathbb{R}$, by considering the first time the process hits 0 and using the strong Markov property, and also with arbitrary level-sets $K^{y}=\left\{s \geq 0: X_{s}=\right.$ $y\}$ and $K_{t}^{y}=K^{y} \cap[0, t], t \geq 0, y \in \mathbb{R}$, by considering the first time the process hits $y$.

Remark. Statement (4.2) is not new. In the case of Brownian motion, which is stable with $\alpha=2$, it was already known to P. Levy, as can be deduced from section 2.2 of Ito and McKean ([11]); in particular, it follows directly from 5) and 6) on page 43, reference [11]. The general case $1<\alpha \leq 2$, follows similarly from the results in section 7 of Fristedt and Taylor ([9]). Furthermore, the results in [9] imply that the limit $L_{t}$ is a constant multiple of the local time at 0 .

One can actually deduce a stronger statement than the a.s. convergence in (4.2) from Theorem 3.1

Theorem 4.1. Let $\left\{X_{t}: t \geq 0\right\}$ be a real-valued stable process, of index $\alpha \in(1,2]$. Then, there exists a set of probability one, on which $\epsilon^{-1 / \alpha}\left|K_{t}(\epsilon)\right|$ converges, as $\epsilon \rightarrow 0$, simultaneously for all $t \geq 0$. Furthermore, the limit $L_{t}, t \geq 0$, is continuous in $t$.

Remark. It is plain that, given its existence, the limit $\left\{L_{t}: t \geq 0\right\}$ is adapted and constitutes an additive functional. As such, and being continuous, it must be a constant multiple of the local time of $X$ at 0 , by Proposition IV.5 of reference 2]. Reversing the point of view, one can define the local time process by $L_{t}=$ $\lim _{\epsilon \rightarrow 0} \epsilon^{-1 / \alpha}\left|K_{t}(\epsilon)\right|, t \geq 0$, without a priori knowledge of its existence.

Proof of Theorem 4.1. Fix $t>0$ and consider the bridge $Y_{s}=(\tau / t)^{-1 / \alpha} X_{s \tau / t}$, $0 \leq s \leq t$, its zero-set $K_{Y}$ and set $K_{Y, s}=K_{Y} \cap[0, s]$. In the notation of subsection 3.1 , we have that $\mathcal{G}_{n}=\{1,2\}^{n}$, for all $n \geq 0$, and $\mathcal{P}=\bigcup_{n=0}^{\infty}\{1,2\}^{n}$. Furthermore, since $\mathcal{P}$ is countable, the limit

$$
L_{Y}^{x}=\lim _{\epsilon \rightarrow 0} \epsilon^{-1 / \alpha}\left|K_{Y}^{x}(\epsilon)\right|
$$

exists for all $x \in \mathcal{P}$ simultaneously on a set $\Omega_{t}^{\prime}$ of probability one, and one has the following equality, for each $x \in \mathcal{P}$ and all $n \geq 0$ :

$$
e^{-D S_{x}} L_{Y}^{x}=\sum_{y \in \mathcal{G}_{n}^{x}} e^{-D S_{x y}} L_{Y}^{x y},
$$

with $D=1-1 / \alpha$. Define random functions $\left\{L_{Y, s}^{(n)}: 0 \leq s \leq t\right\}$, by letting $L_{Y, .}^{(n)}$ be the distribution function of the measure that spreads mass $e^{-D S_{x}} L_{Y}^{x}$ uniformly on the interval $\phi_{x}([0, t])$, for $x \in \mathcal{G}_{n}$. By (4.6) then $L_{Y, t}^{(n)}=L_{Y}$, for all $n \geq 0$.

It is plain that (see Figure 4)

$$
\left\|L_{Y, .}^{(n+1)}-L_{Y, .}^{(n)}\right\|_{\infty} \leq \max _{x \in \mathcal{G}_{n}} e^{-D S_{x}} L_{Y}^{x}
$$

for all $n \geq 0$. We next note the following lemma, whose proof we postpone to the end of this section.

\section{Lemma 4.2 .}

$$
\sum_{n=0}^{\infty} \mathbb{E}\left[\max _{x \in \mathcal{G}_{n}} e^{-D S_{x}} L_{Y}^{x}\right]<\infty
$$




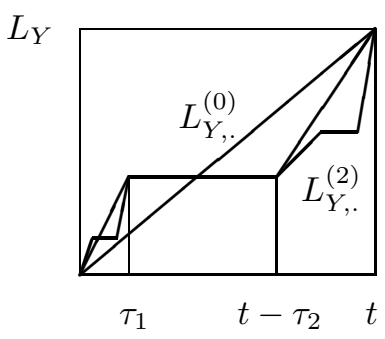

Figure 4 . The functions $L_{Y, .}^{(0)}, L_{Y, .}^{(1)}$ and $L_{Y, .}^{(2)}$.

It now follows from the lemma that on a set $\Omega_{t} \subseteq \Omega_{t}^{\prime}$, of probability one, the functions $L_{Y, \text {. }}^{(n)}$ converge uniformly to a limit $L_{Y, \text {, }}$, which must be continuous. Because $\left|K_{Y, r}(\epsilon)\right| \leq\left|K_{Y, s}(\epsilon)\right|$, for $0 \leq r<s \leq t$, because the limit $L_{Y, \text {. }}$ is continuous and because, by (4.5),

$$
\lim _{\epsilon \rightarrow 0} \epsilon^{-1 / \alpha}\left|K_{Y, s}(\epsilon)\right|=L_{Y, s}
$$

for those $s \in[0, t]$ which are endpoints of the intervals $\phi_{x}([0, t]), x \in \mathcal{P}$, and because such $s$ are dense in $K_{Y}$, it follows that the convergence (4.8) holds simultaneously for all $s \in K_{Y}$. Finally, because both sides of (4.8) are constant functions of $s$ on each of the intervals in $[0, t] \backslash K_{Y}, 4.8$ holds for all $s \in[0, t]$ simultaneously on $\Omega_{t}$.

Using the representation (4.3) (which now holds pointwise and not just in law, by our choice of $Y$ ), we then have that, on $\Omega_{t}$,

$$
L_{s}:=\left(\frac{\tau}{t}\right)^{1-1 / \alpha} L_{Y, s \tau / t}=\lim _{\epsilon \rightarrow 0} \epsilon^{-1 / \alpha}\left|K_{s}(\epsilon)\right|
$$

for all $0 \leq s \leq \tau$; defining $L_{s}=L_{\tau}$, for $\tau<s \leq t$, one has that

$$
\epsilon^{-1 / \alpha}\left|K_{s}(\epsilon)\right| \longrightarrow L_{s}
$$

for all $s \in[0, t]$ on $\Omega_{t}$. Finally, the set $\Omega_{\infty}:=\bigcap_{n \geq 1} \Omega_{n}$ has probability one and on it (4.9) holds for all $t \geq 0$ and the function $t \mapsto L_{t}$ is continuous.

Proof of Lemma 4.2. First note that $e^{-D S_{x}} \leq 2^{-n(1-1 / \alpha)}$ for $x \in \mathcal{G}_{n}$. Hence

$$
\mathbb{E}\left[\max _{x \in \mathcal{G}_{n}} e^{-D S_{x}} L_{Y}^{x}\right] \leq 2^{-n(1-1 / \alpha)} E_{2^{n}},
$$

where $E_{n}$ denotes the expectation of the maximum of $n$ independent copies of $L_{Y}$. We will show that, for any $\epsilon>0$, there exists a constant $0<C_{\epsilon}<\infty$, such that $E_{n} \leq C_{\epsilon} n^{\epsilon}, n \geq 0$, and this, in conjunction with (4.10), will prove the lemma.

Recall the martingale $M_{n}=\sum_{x \in \mathcal{G}_{n}} e^{-D S_{x}}$ and note that, since $M_{1} \leq 2$, its limit $M_{\infty}$ has finite moments of all orders, by Theorem 2.1 of reference [20]. Recall also, from (3.12), that $L_{Y}=M_{\infty} \times$ const.; therefore $L_{Y}$ has finite moments of all orders. It follows from Markov's inequality that, for any $\beta>0$ and $u>0$,

$$
\mathbb{P}\left(L_{Y}>u\right) \leq u^{-\beta} \mathbb{E}\left[L_{Y}^{\beta}\right] .
$$


Fix $\beta>1$ and let $c=\mathbb{E}\left[L_{Y}^{\beta}\right]^{1 / \beta}$. Since $E_{n}=\int_{0}^{\infty}\left\{1-\left[1-\mathbb{P}\left(L_{Y}>u\right)\right]^{n}\right\} d u$,

$$
E_{n} \leq c+\int_{c}^{\infty}\left[1-\left(1-c^{\beta} u^{-\beta}\right)^{n}\right] d u=c\left[1+\beta^{-1} \int_{0}^{1} \frac{1-u^{n}}{(1-u)^{1+1 / \beta}} d u\right]
$$

Using the beta-function,

$$
\int_{0}^{1} \frac{1-u^{n}}{(1-u)^{1+1 / \beta}} d u=\sum_{i=1}^{n} \int_{0}^{1} u^{i-1}(1-u)^{-1 / \beta} d u=\sum_{i=1}^{n} \frac{\beta}{i \beta-1} \prod_{j=1}^{i-1} \frac{j \beta}{j \beta-1},
$$

where, by convention, $\prod_{i=1}^{0}=1$. Now, use the well known relation between sums and products, to obtain

$$
\int_{0}^{1} \frac{1-u^{n}}{(1-u)^{1+1 / \beta}} d u \leq C \sum_{i=2}^{n} \frac{\beta}{i \beta-1}[(i-1) \beta-1]^{1 / \beta}+\frac{\beta}{\beta-1} \leq C^{\prime} n^{1 / \beta},
$$

for some finite constant $C^{\prime}$, which, together with (4.11), prove the lemma.

\section{Concluding Remarks}

1. As should be clear from Example 4.1 (Cantor set), formulae (22.8) and (22.9) can be used for explicit computations, at least in low dimensions $(d=1,2$ say).

2. We conjecture that the non-lattice condition of Theorem 2.3 (i) is also necessary for $\epsilon^{-(d-D)}|K(\epsilon)|$ to converge to a limit as $\epsilon \rightarrow 0$, at least in non-trivial cases. To be more precise, let $I$ denote the convex hull of $K$.

Conjecture. If

$$
I \backslash \bigcup_{i=1}^{N} \phi_{i}(I) \neq \emptyset
$$

then $\epsilon^{-(d-D)}|K(\epsilon)|$ converges to a limit, as $\epsilon \rightarrow 0$, iff

$$
\left\{\log r_{1}, \ldots, \log r_{N}\right\} \nsubseteq \lambda \mathbb{Z} \quad \text { for any } \lambda>0 .
$$

Notice that (5.1) is not satisfied for the set of similarities $\phi_{1}(x)=x / 3, \phi_{2}(x)=$ $(x+1) / 3, \phi_{3}(x)=(x+2) / 3$. The invariant set $K$ for these similarities is $K=[0,1], D=1$, and obviously $\epsilon^{-(1-D)}|K(\epsilon)| \longrightarrow 1$, as $\epsilon \rightarrow 0$. For specific cases of 'lattice self-similar sets', one can verify the conjecture as in Example 4.1 , but it would be nice to have a general argument. (Note added in proof: See also the recent article [26] by van Frankenhuysen and Lapidus; Remark 5.2 there verifies the conjecture for a certain class of self-similar subsets of $\mathbb{R}$.)

3 . The methods of this paper rely heavily on the self-similarity of $K$, i.e., the fact that each of the maps $\phi_{i}$ is a similarity transformation. One can consider the same problem for fractals with some approximate self-similarity, like limit sets of Fuchsian groups and Schottky groups, or hyperbolic Julia sets of rational maps. The problem is considerably harder for such sets, but similar methods apply. 


\section{APPENDIX}

In subsection 3.1 we considered random elements $\Phi=\left\{\phi_{1}, \ldots, \phi_{\nu}\right\}$, defined on some probability space $(\Omega, \mathcal{F}, \mathbb{P})$. Here we consider matters of measurability.

Recall that $\mathcal{S}$ stands for the set of all contracting similarities of $\mathbb{R}^{d}$ and $J$ is a fixed compact set, with $G=\operatorname{int}(J)$ and $\bar{G}=J$. Set $\mathcal{S}_{J}:=\{s \in \mathcal{S}: s(J) \subseteq J\}$ and, for $n>1$

$$
\mathcal{S}_{J}^{(n)}:=\left\{\left\{s_{1}, \ldots, s_{n}\right\}: s_{i}(J) \subseteq J \text { for all } i \text { and } s_{i}(G) \cap s_{j}(G)=\emptyset \text { for } i \neq j\right\} .
$$

Then $\Phi$ takes values in $\mathcal{S}_{J}^{*}:=\bigcup_{n=0}^{\infty} \mathcal{S}_{J}^{(n)}$, where $\mathcal{S}_{J}^{(1)}=\mathcal{S}_{J}$ and $\mathcal{S}_{J}^{(0)}=\{\mathrm{e}\}$.

Each element of $\mathcal{S}$ is of the form $s(v)=r A v+b$, with $r \in(0,1), A \in O\left(\mathbb{R}^{d}\right)$ and $b \in \mathbb{R}^{d}$. We may therefore endow $\mathcal{S}$ with a metric:

$$
\operatorname{dist}\left(s, s^{\prime}\right):=\left|r-r^{\prime}\right|+\left\|A-A^{\prime}\right\|+\left|b-b^{\prime}\right| .
$$

The resulting metric space is separable and complete and $\mathcal{S}_{J}$ is closed in it. We may also endow each $\mathcal{S}^{(n)}$, the set of all subsets of $\mathcal{S}$ with $n$ elements, with a metric, namely

$$
\operatorname{dist}\left(S, S^{\prime}\right)=\min _{\pi} \max _{s \in S} \operatorname{dist}(s, \pi(s)),
$$

where the minimum is taken over all bijections $\pi: S \rightarrow S^{\prime}$. Then, for each $n \geq 1$, $\mathcal{S}_{J}^{(n)}$ is closed in $\mathcal{S}^{(n)}$ and hence in $\mathcal{B}^{(n)}$, the Borel $\sigma$-algebra of $\mathcal{S}^{(n)}$. Therefore $\mathcal{B}_{J}^{(n)}:=\left\{\mathcal{S}_{J}^{(n)} \cap B: B \in \mathcal{B}^{(n)}\right\}$ is a $\sigma$-algebra and we take

$$
\mathcal{B}_{J}^{*}:=\left\{B \in \mathcal{S}^{*}: B \cap \mathcal{S}_{J}^{(n)} \in \mathcal{B}_{J}^{(n)} \text { for all } n \geq 0\right\}
$$

as our $\sigma$-algebra on $\mathcal{S}_{J}^{*}$, where $\mathcal{B}_{J}^{(0)}:=\left\{\emptyset, \mathcal{S}_{J}^{(0)}\right\}$ is the trivial $\sigma$-algebra. We then require of $\Phi$ that it be a map $\Phi: \Omega \rightarrow \mathcal{S}_{J}^{*}$, measurable with respect to $\mathcal{F}$ and $\mathcal{B}_{J}^{*}$.

\section{ADDED AFTER POSTING}

In the Conjecture in section 5, [26] should read [25].

\section{REFERENCES}

1. T. Bedford and A. Fisher, Analogues of the Lebesgue density theorem for fractal sets of reals and integers, Proc. London Math. Soc. 64 (1992), 95-124. MR 92j:58058

2. J. Bertoin, Levy Processes, Cambridge Tracts in Math. 121, Camb. Univ. Press, 1996. MR 98e:60117

3. J. Biggins, Martingale convergence in the branching random walk, J. Appl. Probab. 14 (1977), 25-37. MR 55:6592

4. P. Billingsley, Probability and Measure, 3rd ed., Wiley, New York, 1995. MR 95k:60001

5. R.M. Blumenthal and R.K. Getoor, The dimension of the set of zeros and the graph of a symmetric stable process, Illinois J. Math. 6 (1962), 308-316. MR 25:1581

6. K. Falconer, Random Fractals, Math. Proc. Camb. Phil. Soc. 100 (1986), 559-582. MR 88e:28005

7. 1115-1124. MR 95e:28003

8. W. Feller, An Introduction to Probability Theory and its Applications, Vol. 2, 2nd ed., Wiley, New York, 1971. MR 42:5292

9. B. Fristedt and S.J. Taylor, Constructions of local time for a Markov process, Z. Wahrsch. verw. Gebiete 62 (1983), 73-112. MR 85c:60121

10. J. Hutchinson, Fractals and self-similarity, Indiana Univ. Math. J. 30 (1981), 713-747. MR 82h:49026

11. K. Ito and H. McKean, Diffusion processes and their sample paths, Springer Verlag, BerlinHeidelberg, 1965. MR 33:8031 
12. S. Lalley, The packing and covering functions of some self-similar fractals, Indiana Univ. Math. J. 37 (1988), 699-709. MR 89k:28013

13. _ A renewal theorems in symbolic dynamics, with applications to geodesic flows, noneuclidean tessellations, and their fractal limits, Acta Math. 163 (1989), 1-55. MR 91c:58112

14. M. Lapidus, Fractal drum, inverse spectral problems for elliptic operators and a partial resolution of the Weyl-Berry conjecture, Trans. Amer. Math. Soc. 325 (1991), 465-529. MR 91j:58163

15. M. Lapidus and C. Pomerance, The Riemann zeta-function and the one-dimensional Weyl-Berry conjecture for fractal drums, Proc. London Math. Soc. (3) 66 (1993), 41-69. MR 93k:58217

16. Counterexamples to the modified Weyl-Berry conjecture on fractal drums, Math. Proc. Camb. Phil. Soc. 119 (1996), 167-178. MR 96h:58175

17. R. Lyons, A simple path to Biggins' martingale convergence for branching random walk, in Classical and Modern Branching Processes (K. Athreya, P. Jagers, eds.), IMA Vol. Math. Appl., Vol. 84, Springer Verlag, New York, 1997, pp. 217-221. CMP 98:08

18. R. Lyons, R. Pemantle and Y. Peres, Conceptual proofs of $L \log L$ criteria for mean behavior of branching processes, Ann. Probab. 23 (1995), 1125-1138. MR 96m:60194

19. B. Mandelbrot, The Fractal Geometry of Nature, Freeman, San Francisco, 1983. MR 84h:00021

20. D. Mauldin and S. Williams, Random recursive constructions: Asymptotic geometric and topological properties, Trans. Amer. Math. Soc. 295 (1986), 325-346. MR 87j:60027

21. P. Moran, Additive functions of intervals and Hausdorff measure, Proc. Camb. Phil. Soc. 42 (1946), 15-23. MR 7:278f

22. O. Nerman, On the convergence of supercritical general (C-M-J) branching processes, Z. Wahrsch. verw. Gebiete 57 (1981), 365-395. MR 82m:60104

23. A. Schief, Separation properties of self-similar sets, Proc. Amer. Math. Soc. 122 (1994), 111-115. MR 94k:28012

24. S.J. Taylor and J.G. Wendel, The exact Hausdorff measure of the zero set of a stable process, Z. Wahrsch. verw. Gebiete 6 (1966), 170-180. MR 35:1090

25. M. Lapidus and M. van Frankenhuysen, Complex dimensions of fractal strings and oscillatory phenomena in fractal geometry and arithmetic, Spectral Problems in Geometry and Arithmetic (T. Branson, ed.), Contemporary Mathematics, Vol. 237, Amer. Math. Soc., 1999.

26. M. Lapidus, Vibrations of fractal drums, the Riemann hypothesis, waves in fractal media, and the Weyl-Berry conjecture, in Ordinary and Partial Differential Equations, Vol. IV, Proc. 12th International Conference on the Theory of Ordinary and Partial Differential Equations (Dundee, Scotland), Pitman Research Notes in Mathematics Series 289 (B. D. Sleeman and R. J. Jarvis Eds.), Longman Scientific and Technical, London, 1993, pp. 126-209. MR 95g:58247

Statistical Laboratory, University of Cambridge, 16 Mill Lane, Cambridge CB2 1SB, ENGLAND, U.K.

Current address: Department of Mathematics, University of Crete, 71409 Iraklion, Crete, Greece

E-mail address: gatzoura@math.uch.gr 